\title{
The First Tidally Disrupted Ultra-faint Dwarf Galaxy?: A Spectroscopic Analysis of the Tucana III Stream*†
}

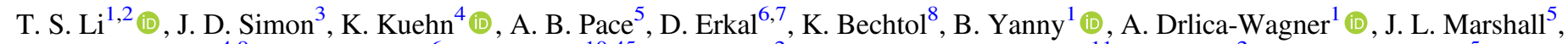
C. Lidman $^{4,9}$ (1), E. Balbinot ${ }^{6}$, D. Carollo ${ }^{10,45}$, S. Jenkins ${ }^{2}$, C. E. Martínez-Vázquez ${ }^{11}$, N. Shipp ${ }^{2}$ (10, K. M. Stringer ${ }^{5}$,

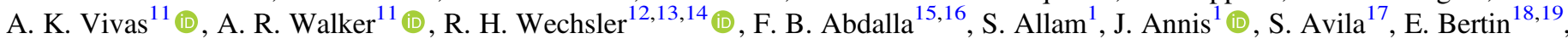
D. Brooks ${ }^{15}$ (D) , E. Buckley-Geer ${ }^{1}$, D. L. Burke ${ }^{13,14}$ (10) A. Carnero Rosell ${ }^{20,21}$, M. Carrasco Kind ${ }^{22,23}$, J. Carretero ${ }^{24}$, C. E. Cunha ${ }^{13}$, C. B. D’Andrea ${ }^{25}$, L. N. da Costa ${ }^{20,21}$, C. Davis ${ }^{13}$, J. De Vicente ${ }^{26}$, P. Doel ${ }^{15}$, T. F. Eifler ${ }^{27,28}$, A. E. Evrard ${ }^{29,30}$, B. Flaugher ${ }^{1}$, J. Frieman ${ }^{1,2}$, J. García-Bellido ${ }^{31}$ (1) E. Gaztanaga ${ }^{32,33}$, D. W. Gerdes ${ }^{29,30}$ (1), D. Gruen ${ }^{13,14}$ (1) R. A. Gruend2 ${ }^{22,23}$, J. Gschwend ${ }^{20,21}$, G. Gutierrez ${ }^{1}$, W. G. Hartley ${ }^{15,34}$, D. L. Hollowood ${ }^{35}$, K. Honscheid ${ }^{36,37}$, D. J. James ${ }^{38}$, E. Krause ${ }^{27,28}$, M. A. G. Maia ${ }^{20,21}$, M. March $^{25}$, F. Menanteau ${ }^{22,23}$, R. Miquel ${ }^{24,39}$, A. A. Plazas ${ }^{28}$, E. Sanchez ${ }^{26}$, B. Santiago ${ }^{20,40}$, V. Scarpine ${ }^{1}$, R. Schindler ${ }^{14}$, M. Schubnell ${ }^{30}$, I. Sevilla-Noarbe ${ }^{26}$, M. Smith ${ }^{41}$, R. C. Smith $^{11}$, M. Soares-Santos ${ }^{42}$ (D) F. Sobreira ${ }^{20,43}$, E. Suchyta ${ }^{44}$, M. E. C. Swanson ${ }^{23}$, G. Tarle ${ }^{30}(1)$, and D. L. Tucker ${ }^{1}$

(DES Collaboration)

\footnotetext{
${ }^{1}$ Fermi National Accelerator Laboratory, P.O. Box 500, Batavia, IL 60510, USA; tingli@fnal.gov

${ }^{2}$ Kavli Institute for Cosmological Physics, University of Chicago, Chicago, IL 60637, USA

${ }^{3}$ Observatories of the Carnegie Institution for Science, 813 Santa Barbara St., Pasadena, CA 91101, USA ${ }^{4}$ Australian Astronomical Observatory, North Ryde, NSW 2113, Australia

${ }^{5}$ George P. and Cynthia Woods Mitchell Institute for Fundamental Physics and Astronomy, and Department of Physics and Astronomy, Texas A\&M University, College Station, TX 77843, USA

${ }^{6}$ Department of Physics, University of Surrey, Guildford GU2 7XH, UK

${ }^{7}$ Institute of Astronomy, University of Cambridge, Madingley Road, Cambridge CB3 0HA, UK

${ }^{9}$ TSST, 933 North Cherry Avenue, Tucson, AZ 85721, USA
Thearch School of Astronomy and Astrophysics, Australian National University, ACT 2601, Australia

${ }^{10}$ INAF Osservatorio Astrofisico di Torino, Via Osservatorio 20, I-10025 Pino Torinese, Italy

${ }^{11}$ Cerro Tololo Inter-American Observatory, National Optical Astronomy Observatory, Casilla 603, La Serena, Chile

${ }_{12}$ Department of Physics, Stanford University, 382 Via Pueblo Mall, Stanford, CA 94305, USA

${ }^{13}$ Kavli Institute for Particle Astrophysics \& Cosmology, P.O. Box 2450, Stanford University, Stanford, CA 94305, USA

${ }^{14}$ SLAC National Accelerator Laboratory, Menlo Park, CA 94025, USA

${ }_{15}^{15}$ Department of Physics \& Astronomy, University College London, Gower Street, London, WC1E 6BT, UK

${ }^{16}$ Department of Physics and Electronics, Rhodes University, P.O. Box 94, Grahamstown, 6140, South Africa

${ }^{7}$ Institute of Cosmology \& Gravitation, University of Portsmouth, Portsmouth, PO1 3FX, UK

${ }^{18}$ CNRS, UMR 7095, Institut d'Astrophysique de Paris, F-75014, Paris, France

${ }^{19}$ Sorbonne Universités, UPMC Univ Paris 06, UMR 7095, Institut d'Astrophysique de Paris, F-75014, Paris, France

${ }^{20}$ Laboratório Interinstitucional de e-Astronomia-LIneA, Rua Gal. José Cristino 77, Rio de Janeiro, RJ-20921-400, Brazil

${ }^{21}$ Observatório Nacional, Rua Gal. José Cristino 77, Rio de Janeiro, RJ-20921-400, Brazil

${ }^{22}$ Department of Astronomy, University of Illinois at Urbana-Champaign, 1002 W. Green Street, Urbana, IL 61801, USA

${ }^{23}$ National Center for Supercomputing Applications, 1205 West Clark St., Urbana, IL 61801, USA

${ }^{24}$ Institut de Física d'Altes Energies (IFAE), The Barcelona Institute of Science and Technology, Campus UAB, E-08193 Bellaterra (Barcelona), Spain

${ }^{25}$ Department of Physics and Astronomy, University of Pennsylvania, Philadelphia, PA 19104, USA

${ }^{26}$ Centro de Investigaciones Energéticas, Medioambientales y Tecnológicas (CIEMAT), Madrid, Spain

${ }^{27}$ Department of Astronomy/Steward Observatory, 933 North Cherry Avenue, Tucson, AZ 85721-0065, USA

${ }^{28}$ Jet Propulsion Laboratory, California Institute of Technology, 4800 Oak Grove Dr., Pasadena, CA 91109, USA

29 Department of Astronomy, University of Michigan, Ann Arbor, MI 48109, USA

${ }^{30}$ Department of Physics, University of Michigan, Ann Arbor, MI 48109, USA

${ }^{31}$ Instituto de Fisica Teorica UAM/CSIC, Universidad Autonoma de Madrid, E-28049 Madrid, Spain

${ }^{32}$ Institut d'Estudis Espacials de Catalunya (IEEC), E-08193 Barcelona, Spain

${ }^{33}$ Institute of Space Sciences (ICE, CSIC), Campus UAB, Carrer de Can Magrans, s/n, E-08193 Barcelona, Spain

${ }^{34}$ Department of Physics, ETH Zurich, Wolfgang-Pauli-Strasse 16, CH-8093 Zurich, Switzerland ${ }^{35}$ Santa Cruz Institute for Particle Physics, Santa Cruz, CA 95064, USA

${ }^{36}$ Center for Cosmology and Astro-Particle Physics, The Ohio State University, Columbus, OH 43210, USA

${ }^{37}$ Department of Physics, The Ohio State University, Columbus, OH 43210, USA

${ }^{38}$ Harvard-Smithsonian Center for Astrophysics, Cambridge, MA 02138, USA

${ }^{39}$ Institució Catalana de Recerca i Estudis Avançats, E-08010 Barcelona, Spain

${ }^{40}$ Instituto de Física, UFRGS, Caixa Postal 15051, Porto Alegre, RS-91501-970, Brazil

${ }^{41}$ School of Physics and Astronomy, University of Southampton, Southampton, SO17 1BJ, UK

${ }^{42}$ Brandeis University, Physics Department, 415 South Street, Waltham, MA 02453, USA

${ }^{43}$ Instituto de Física Gleb Wataghin, Universidade Estadual de Campinas, 13083-859, Campinas, SP, Brazil

${ }^{44}$ Computer Science and Mathematics Division, Oak Ridge National Laboratory, Oak Ridge, TN 37831, USA

Received 2018 April 20; revised 2018 September 3; accepted 2018 September 3; published 2018 October 5
}

\footnotetext{
* This paper includes data gathered with the Anglo-Australian Telescope in Australia.

$\dagger$ This paper includes data gathered with the $6.5 \mathrm{~m}$ Magellan Telescopes located at Las Campanas Observatory, Chile.

45 ARC Centre of Excellence for All-sky Astrophysics (CAASTRO).
} 


\begin{abstract}
We present a spectroscopic study of the tidal tails and core of the Milky Way satellite Tucana III, collectively referred to as the Tucana III stream, using the $2 \mathrm{dF}+\mathrm{AAOmega}$ spectrograph on the Anglo-Australian Telescope and the IMACS spectrograph on the Magellan Baade Telescope. In addition to recovering the brightest nine previously known member stars in the Tucana III core, we identify 22 members in the tidal tails. We observe strong evidence for a velocity gradient of $8.0 \pm 0.4 \mathrm{~km} \mathrm{~s}^{-1} \mathrm{deg}^{-1}$ over at least $3^{\circ}$ on the sky. Based on the continuity in velocity, we confirm that the Tucana III tails are real tidal extensions of Tucana III. The large velocity gradient of the stream implies that Tucana III is likely on a radial orbit. We successfully obtain metallicities for four members in the core and 12 members in the tails. We find that members close to the ends of the stream tend to be more metal-poor than members in the core, indicating a possible metallicity gradient between the center of the progenitor halo and its edge. The spread in metallicity suggests that the progenitor of the Tucana III stream is likely a dwarf galaxy rather than a star cluster. Furthermore, we find that with the precise photometry of the Dark Energy Survey data, there is a discernible color offset between metal-rich disk stars and metal-poor stream members. This metallicity-dependent color offers a more efficient method to recognize metal-poor targets and will increase the selection efficiency of stream members for future spectroscopic follow-up programs on stellar streams.
\end{abstract}

Key words: dark matter - galaxies: dwarf - galaxies: individual (Tucana III) - Local Group - stars: abundances

Supporting material: machine-readable tables

\section{Introduction}

Stellar streams, originating from the tidal disruption of dwarf galaxies (e.g., the Sagittarius stream; Majewski et al. 2003; Belokurov et al. 2006b) and globular clusters (e.g., the Palomar 5 tidal tails; Odenkirchen et al. 2001), are excellent tracers for probing the underlying shape of the Milky Way's (MW) dark matter halo (see, e.g., Johnston et al. 2005; Law \& Majewski 2010; Bonaca et al. 2014; Bovy 2014; Küpper et al. 2015; Bovy et al. 2016; Erkal et al. 2016). Density perturbations along kinematically cold (i.e., small velocity dispersion) stellar streams can additionally be used to measure the abundance of low-mass dark matter substructure (e.g., Ibata et al. 2002; Johnston et al. 2002; Carlberg 2009, 2016; Yoon et al. 2011; Erkal \& Belokurov 2015a; Bovy et al. 2017; Erkal et al. 2017) and therefore provide a direct test of the standard $\Lambda \mathrm{CDM}$ cosmological model (Springel et al. 2008). These diffuse stellar features also inform us that our Galactic stellar halo is shaped by the merging of neighboring smaller galaxies, which is predicted by hierarchical structure formation models of galaxy evolution (Peebles 1965; Searle \& Zinn 1978; Steinmetz \& Navarro 2002; Bullock \& Johnston 2005; Font et al. 2011). We refer readers to Newberg \& Carlin (2016) for a recent review.

Thanks to the unprecedented photometric precision, depth, and coverage area of the Dark Energy Survey (DES; DES Collaboration 2005, 2016, 2017; Abbott et al. 2018), more than a dozen new stellar streams have been discovered using the first $3 \mathrm{yr}$ of data (Drlica-Wagner et al. 2015; Balbinot et al. 2016; Shipp et al. 2018). Of the streams discovered in the DES, the Tucana III (Tuc III) stellar stream is among the most intriguing because it is the only new stream that has an unambiguous progenitor identified.

The Tuc III stellar stream was discovered as a pair of very low surface brightness linear features adjacent to the ultra-faint dwarf galaxy candidate Tuc III (Drlica-Wagner et al. 2015). Located at a heliocentric distance of just $\sim 25 \mathrm{kpc}$ and a Galactocentric distance of $\sim 23 \mathrm{kpc}$, the stream extends at least $2^{\circ}$ from either side of Tuc III. Further analyses conducted by Shipp et al. (2018) showed that the stream has a projected length of $2 \mathrm{kpc}(4.8)$ and a width of $79 \mathrm{pc}\left(\sigma_{w}=0.18\right)$ on the sky. The stream is the result of the ongoing disruption of Tuc III. Following the terminology that was used in Shipp et al. (2018), in this paper, we will refer to the Tuc III dwarf galaxy (candidate) as the Tuc III core, the tidal tails of Tuc III as the Tuc III tails, and the whole system as the Tuc III stream. Specifically, we consider all Tuc III member stars confirmed in Simon et al. (2017) as belonging to the Tuc III core. We define the radius of the Tuc III core $r$ to be the radial distance to the outermost Tuc III member confirmed in Simon et al. (2017), i.e., a radius of $r \lesssim 0.22$ (or $\sim 97 \mathrm{pc}$ ), or 2.2 times the half-light radius of the Tuc III dwarf galaxy candidate determined in DrlicaWagner et al. (2015).

If Tuc III is a dwarf galaxy, the Tuc III stream will be a prototype for the tidal disruption of the smallest galaxies. Spectroscopic observations were conducted in the Tuc III core by Simon et al. (2017) with Magellan/IMACS, but the authors were not able to conclusively classify Tuc III as an ultra-faint dwarf galaxy or star cluster due to the unresolved velocity and metallicity dispersions. Simon et al. (2017) tentatively suggested that Tuc III is the tidally stripped remnant of a dark matter-dominated dwarf galaxy, based on its large size and low mean metallicity.

Though dozens of streams have been found in the MW halo, only a handful of streams have an unambiguous progenitor identified (e.g., the Sagittarius stream, Palomar 5 tidal tails, and NGC 5466 tidal tails; Belokurov et al. 2006a). The Tuc III stream offers an opportunity to investigate mechanisms of tidal disruption and the resulting formation of tidal streams in great detail. Furthermore, the presence of the progenitor also makes the stream an ideal candidate for orbit fitting, as well as a valuable tracer of the MW's gravitational potential.

In this paper, we present results from spectroscopic observations of the Tuc III stream using the $2 \mathrm{dF}+\mathrm{AAOmega}$ spectrograph on the Anglo-Australian Telescope (AAT) and the IMACS spectrograph on the Magellan Baade Telescope. We describe the observations and data reduction from these two instruments in Section 2 . We identify member stars of the stream and determine the kinematic and metallicity properties of the stream in Section 3. In Section 4, we discuss the properties of the stream and the comparison with other known streams and dwarf galaxies. In Section 5, we demonstrate the use of photometric measurements to select candidate metal-poor stream members based on their colors for future spectroscopic follow-up programs. We conclude in Section 6.

\section{Observations and Data Reduction}

\subsection{AAT/2df+AAOmega Observations}

We observed candidate member stars in the Tuc III stream with the AAOmega spectrograph (Sharp et al. 2006) on the 
$3.9 \mathrm{~m}$ AAT. AAOmega is a dual-beam spectrograph that feeds a blue arm and a red arm with a beam splitter at $5700 \AA$. This paper focuses on the spectra obtained with the red arm using the 1700D grating, which has a spectral resolution of $R=10,000$, a pixel scale of $0.23 \AA$ pixel $^{-1}$, and a wavelength coverage of $8400-8810 \AA$. This wavelength range contains the calcium triplet $(\mathrm{CaT})$ absorption lines that are the primary source of velocity and metallicity measurements.

The AAOmega spectrograph is fed by the Two Degree Field (2dF) fiber positioner facility, allowing the acquisition of up to 400 simultaneous spectra of objects within a $2^{\circ}$ field in diameter on the sky. Among the 400 fibers, 25 are assigned to sky positions, and eight are assigned to guide stars selected from the UCAC4 catalog (Zacharias et al. 2013). The remaining fibers are assigned to the target stars.

We selected the targets using an empirical color-magnitude locus derived from the confirmed member stars in the Tuc III dwarf galaxy (Simon et al. 2017). Based on the location of candidate stars on the sky and color-magnitude diagram using an early version of DES astrometry and photometry, namely the Y2Q catalog (Drlica-Wagner et al. 2015), a membership probability for each star was calculated in a similar way as discussed in Drlica-Wagner et al. (2015). We then prioritized targets based on a combination of brightness and membership probability and allocated targets to the fibers using the configure $^{46}$ software provided by the Australian Astronomical Observatory. As shown in Figure 1, we selected several hundred main-sequence turnoff (MSTO) and red giant branch (RGB) stars, a few dozen red horizontal branch (RHB) stars, and a handful of blue horizontal branch (BHB) stars.

Observations were conducted on 2016 June 30, July 9-13, and July 25-27 and 2017 August 22. We had a total of nine half nights of observing time in 2016 and $2 \mathrm{hr}$ of service time in 2017. The exposures are typically composed of several 30-40 minute exposures. Due to unfavorable weather conditions, we did not observe on July 11 . The dome was partially closed for many nights due to clouds, including June 30 and July 9, 13, 26, and 27. Exposures on July 12 and 13 were taken through thick clouds and were therefore excluded from the analysis. About half of the exposures taken on July 10 were also discarded due to the presence of thin clouds. Specifically, data are excluded from the analysis when the extracted 1D spectra from one exposure have signal-to-noise ratios (S/Ns) of less than 2 pixel $^{-1}$ for stars at $g \sim 18$. As a comparison, with a 30 minute exposure, the $\mathrm{S} / \mathrm{N}$ is around $7 \mathrm{pixel}^{-1}$ for stars at $g \sim 18$ in the absence of clouds. Overall, we lost roughly six of the nine half nights due to poor weather. The remaining useful three half nights have an average seeing of $\sim 2$." 5 .

Since the length of the stream was determined to be about $4^{\circ}$ at the time of discovery (Drlica-Wagner et al. 2015), we targeted the Tuc III stream with two telescope pointings whose centers are offset by 0.8 west (AAT-Field-1) and east (AAT-Field-2) from the center of the Tuc III core during the 2016 classical observing time. This ensures that the Tuc III core will get longer exposures in total and may reveal more faint members. Subsequent reanalysis by Shipp et al. (2018) revealed that the stream is slightly longer (4.8), and we therefore added a third pointing (AAT-Field-3) 1.2 west from the center of Tuc III for $2 \mathrm{hr}$ of observing on 2017 August 22 using service time. Due to the shorter exposure time, AAT-Field-3 is shallower than the

\footnotetext{
46 configure provides a graphic user interface for fiber allocations; see details at https://www.aao.gov.au/science/software/configure.
}

other two pointings. An illustration of the three fields on the sky along with the targets observed is shown in the top left panel of Figure 1 . We obtained $\mathrm{S} / \mathrm{N} \sim 15$ pixel $^{-1}$ for stars at $g \sim 18$ in AAT-Field-1 and AAT-Field-2 and S/N $\sim 9$ pixel $^{-1}$ for stars at $g \sim 18$ in AAT-Field-3. Though the $2 \mathrm{df}$ has a total of 400 fibers (including 25 sky fibers and eight guide stars) and a fiber collision radius of $30^{\prime \prime}-40^{\prime \prime}$, flexibility in fiber allocation with $2 \mathrm{dF}$ allows us to change the targets from night to night and observe more targets at each field. Specifically, we performed quick data reduction and analysis on the observed spectra at the end of each night, measured the radial velocities (RVs) of bright stars whose spectra have sufficient $\mathrm{S} / \mathrm{Ns}(\mathrm{S} / \mathrm{N}>10)$, and classified those stars that have velocity differences more than $100 \mathrm{~km} \mathrm{~s}^{-1}$ from the Tuc III core velocity as nonmembers. We then reallocated the fibers for those nonmembers to alternate targets in the subsequent night's observing. A total of 1045 candidate stars were observed over the entire program with AAT in three fields. We observed roughly $85 \%$ of the RGB candidates at $g<19.5$ in the fields of three AAT pointings; the other $15 \%$ of the candidates were not observed due to either fiber collision or low membership probability of the targets and the limited number of available fibers. We expect that the completeness of the true members at $g<19.5$ is higher than $85 \%$ because the stars that are closer to the empirical color-magnitude locus will have a higher membership probability and therefore a higher priority to be assigned to a fiber.

The data reduction was performed using version 6.46 of $2 d f d r .{ }^{47}$ The reduction includes bias subtraction, 2D scatteredlight subtraction, flat fielding, Gaussian weighted spectral extraction, wavelength calibration, and sky subtraction. Wavelength calibration was first performed using the arc frames taken immediately before or after each science exposure, followed by a recalibration with a second-order polynomial fit using sky emission lines. As the observations were taken from different nights, the reduced and extracted spectra were first corrected for the heliocentric motion of the Sun at each exposure. Then the spectra from multiple exposures were combined using inverse-variance weighting. The final velocities and metallicities reported (see Section 2.3) are derived from the combined spectra over the entire program.

\subsection{Magellan/IMACS Observations}

In order to better probe the transition region $(0.3 \lesssim r \lesssim 0.5)$ between the (presumably) bound core of Tuc III and the tidal tails, we also obtained additional spectroscopy of Tuc III with the IMACS spectrograph (Dressler et al. 2006) on the Magellan Baade telescope. We observed three slit masks on the nights of 2017 June 19 and 21. Target selection for these masks followed the criteria described by Simon et al. (2017), and the mask positions were chosen based on the highest densities of bright candidate RGB stars. The spectrograph configuration was identical to those used by $\mathrm{Li}$ et al. (2017) and Simon et al. (2017), with the $f / 4$ camera and the $1200 / 32.7$ grating providing a spectral resolution of $R=11,000$ over the wavelength range $\sim 7500-8800 \AA$. Observing conditions on June 19 for the first two masks were good (clear skies and seeing $<0$ ". 8 ), while the third mask on June 21 suffered from quite poor conditions (clouds and seeing $>1^{\prime \prime}$ ). Two of the masks, which were both offset from the stream track, did not reveal any additional member stars, while the mask on the stream track identified two new members (both much fainter than what AAT can detect; see top right panel of

\footnotetext{
47 https://www.aao.gov.au/science/software/2dfdr
} 

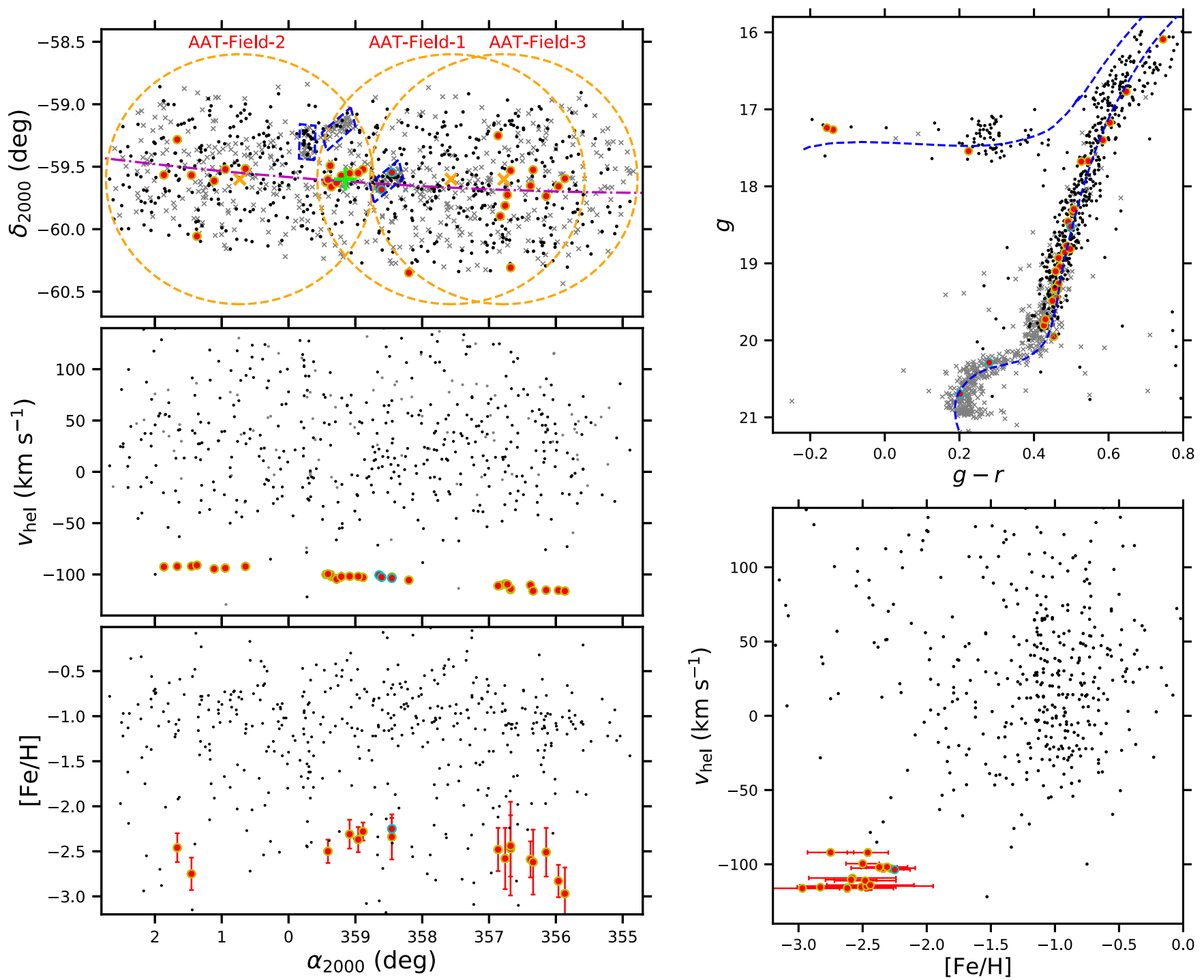

Figure 1. Top left: spatial distribution of the target stars in celestial coordinates $\left(\alpha_{2000}, \delta_{2000}\right)$. The center of the Tuc III dwarf galaxy is indicated with a green plus sign. The three orange crosses indicate the center of the three AAT pointings along with the three orange circles to illustrate the field of view of AAT. The blue dashed boxes show the locations of three IMACS pointings. The magenta dashed line shows a great circle on the sky using the end points of the Tuc III stream from Shipp et al. (2018). The gray crosses represent the targets for which RV measurements failed, while the black dots are the stars whose spectra have high enough $\mathrm{S} / \mathrm{N}$ $(\mathrm{S} / \mathrm{N} \gtrsim 6)$ for $\mathrm{RV}$ measurements. The red filled circles are the confirmed spectroscopic members of the Tuc III stream from this work, with AAT members encircled in yellow and IMACS members encircled in cyan. (Symbols are the same for other panels and other figures if not specified.) Top right: location of target stars in the color-magnitude diagram. The blue lines show a Dotter isochrone (Dotter et al. 2008) for MSTO+RGB stars and a PARSEC isochrone (Bressan et al. 2012) for horizontal-branch stars, both with $[\mathrm{Fe} / \mathrm{H}]=-2.2$ and age $=12.5 \mathrm{Gyr}$ at distance modulus $m-M=17.0$. Middle left: measured heliocentric RV of all targets. Thirty-one member stars of the Tuc III stream are grouped around $-100 \mathrm{~km} \mathrm{~s}^{-1}$. A clear trend of increasing velocity toward equatorial east (or larger $\alpha$ ) is evident from the spectroscopically confirmed members. The uncertainties on the velocities are smaller than the symbol sizes of the member stars. Black (gray) dots represent the stars for which the metallicity measurements are successful (unsuccessful) and are (are not) shown in the bottom panel. Bottom left: subset of the spectra having measured metallicities derived from the EWs of CaT lines (assuming these stars are at the distance of Tuc III). The metallicities of 16 member stars in the Tuc III stream are successfully obtained. The metallicities of the members at the edges of the stream seem to be more metal-poor than the other members in the core of the stream. Bottom right: scatter plot of the measured heliocentric velocity and metallicity of all the targets. The confirmed members are grouped around $[\mathrm{Fe} / \mathrm{H}] \sim-2.5$ and $v_{\text {hel }} \sim-100 \mathrm{~km} \mathrm{~s}^{-1}$. Note that for better display, the error bars in $[\mathrm{Fe} / \mathrm{H}]$ and $v_{\text {hel }}$ are only shown for member stars. The error bars for the target stars are at a similar level as those for the member stars.

Figure 1). These results suggest that Tuc III members at these radii are concentrated in the unbound tails, and relatively few bright members of the core remain to be found.

The IMACS data were reduced as described by Simon et al. (2017) and Li et al. (2017), employing a combination of the Cosmos reduction pipeline (Dressler et al. 2011; Oemler et al. 2017) and a version of the DEEP2 data reduction pipeline (Cooper et al. 2012; Newman et al. 2013) adapted for IMACS.

\subsection{Velocity and Metallicity Measurements}

The reduced 1D spectra from AAT/2df+AAOmega (hereafter AAT) and Magellan/IMACS (hereafter IMACS) were then used for RV measurements following the same method as 
described in Li et al. $(2017,2018)$ and metallicity measurements following Simon et al. (2015) and Li et al. (2017). We refer readers to these works for more details, and we briefly summarize the procedures below.

The RVs were measured via template fitting using a set of RV standards (with various metallicities, temperatures, and surface gravities) observed with the same instrument setup and a maximum-likelihood approach with a Markov chain Monte Carlo (MCMC) sampler. The statistical velocity uncertainties were determined from the standard deviation of the posterior velocity distribution from the MCMC sampler. We adopted a systematic floor of $0.5 \mathrm{~km} \mathrm{~s}^{-1}$ for AAT velocities (Li et al. 2018) and $1.0 \mathrm{~km} \mathrm{~s}^{-1}$ for IMACS velocities (Li et al. 2017; Simon et al. 2017) and added these systematic uncertainties in quadrature with the statistical uncertainties for each star to obtain the final reported velocity uncertainties.

We also determined the metallicities of RGB candidate stars using the equivalent widths (EWs) of the CaT lines. We fit all three of the CaT lines with a Gaussian-plus-Lorentzian function and then converted the summed EWs of the three CaT lines to metallicity using the calibration relation as a function of absolute $V$ magnitude from Carrera et al. (2013). We assume that the candidate stars are members of the Tuc III stream and are therefore at a distance of $25 \pm 2 \mathrm{kpc}$ (Drlica-Wagner et al. 2015) to derive the absolute magnitude of each candidate star. The uncertainties on the EWs are calculated from the Gaussian and Lorentzian fit plus a systematic uncertainty of $0.2 \AA$ ( $\mathrm{Li}$ et al. 2017,2018 ) added in quadrature. The metallicity uncertainties are dominated by the uncertainties on the CaT EWs, with small contributions from the uncertainties on the distances, the stellar photometry, and the uncertainties on the calibration parameters from Carrera et al. (2013). We note that Shipp et al. (2018) found that the Tuc III stream spans $8 \mathrm{kpc}$ in distance. A $4 \mathrm{kpc}$ shift from $25 \mathrm{kpc}$ will cause roughly a $0.05 \mathrm{dex}$ shift in $[\mathrm{Fe} / \mathrm{H}]$ and is small compared to the uncertainties from the EW measurement (typically at $0.25 \mathrm{dex}$ ). We therefore excluded the possible distance gradient when computing the metallicity of member stars in the Tuc III stream.

We applied the methods described above to the entire spectroscopic sample and report the derived RVs and metallicities in Table 1 . We note that not all spectra have high enough $\mathrm{S} / \mathrm{Ns}$ for adequate RV and EW fits. We assess the fitting quality of every spectrum visually. Usually, spectra that have $\mathrm{S} / \mathrm{N}<6$ do not provide a good RV fit, and spectra that have $\mathrm{S} / \mathrm{N}<9$ do not provide a good EW fit. In total, we successfully determined the RVs of 552 candidate stars and EWs of 431 candidate stars from AAT. For IMACS, we determined the RVs of 57 candidates and EWs of 35 candidates. In total, 13 spectra with $\mathrm{S} / \mathrm{N}>6$ were rejected because of a poor RV fit, while 29 spectra with $\mathrm{S} / \mathrm{N}>9$ were rejected because of a poor EW fit. We note that derived metallicities are only valid for stars that are truly RGB members of the Tuc III stream. For the nonmembers, they are likely foreground main-sequence stars from the MW at a different distance, so the calibration relation from Carrera et al. (2013) does not apply to these stars.

We note that even though we used the DES Y2Q catalog for target selection, the reported astrometry and photometry in Table 1 are from a newer version, namely the DES DR1 catalog (Abbott et al. 2018), and are used for analysis in later sections. Specifically, the weighted average magnitudes (WAVG_MAG_PSF) from DR1 are used throughout this work. The improved photometry precision in DES DR1 is especially important for the later discussion in Section 5. However, we noticed that $\sim 2 \%$ of the candidate stars present in the DES Y2Q catalog are missing in DES DR1. This is due to an overly conservative rejection of stars that lie in the wings of nearby saturated stars when the weighted average magnitude (WAVG_ MAG_PSF) quantities in DR1 are computed. For those stars, we present their photometry from the Y2Q catalog and mark them in Table 1. We note that the magnitudes reported here (and used in the analysis in later sections) are all dereddened. For the DR1 catalog, the correction is applied using the $E(B-V)$ values from the reddening map of Schlegel et al. (1998) and extinction coefficient $R_{b}$ derived using the Fitzpatrick (1999) reddening law and Schlafly \& Finkbeiner (2011) adjusted reddening normalization parameter; for the $\mathrm{Y} 2 \mathrm{Q}$ catalog, the correction is made using the stellar locus regression. Details on the reddening corrections can be found from the corresponding references: Abbott et al. (2018) for DR1 and Drlica-Wagner et al. (2015) for Y2Q.

\section{Results}

In this section, we describe the new stream members identified from this work, from which we derived the velocity gradient of the stream, as well as the metallicity dispersion. We also compared our measurements and results with those from Simon et al. (2017) for the members in the Tuc III core.

\subsection{Spectroscopic Membership Determination}

We identified a total of 31 members in the AAT and IMACS combined data set, nine of which are members of the Tuc III core and were previously confirmed in Simon et al. (2017). The other 22 members are in the Tuc III tails and are identified for the first time in this work. Adding the 26 members from the Tuc III core from Simon et al. (2017), the total sample in the Tuc III stream increases to 48 stars. As shown in Figure 1, the member stars form a clear peak around $v_{\text {hel }} \sim-100 \mathrm{~km} \mathrm{~s}^{-1}$ and $[\mathrm{Fe} / \mathrm{H}] \sim-2.5$. Though a few candidate stars are at a similar velocity or metallicity if we only consider one property or the other, combining the two quantities separates the Tuc III members from nonmembers, as shown in the bottom right panel of Figure 1.

We note that the membership identification of Tuc III stream members is a subjective selection process using the following parameters: velocity, metallicity, color, magnitude, and the spectrum itself. Specifically, we examined the candidate stars that have velocities between -140 and $-70 \mathrm{~km} \mathrm{~s}^{-1}$ star-by-star and listed their parameters in Table 1 . Since the bulk velocity of the Tuc III stream $\left(\sim-100 \mathrm{~km} \mathrm{~s}^{-1}\right)$ is far away from that of the MW disk stars, the MW foreground contamination in this velocity range is minimal. The membership of the Tuc III stream is mostly unambiguous, especially through its location on the velocity versus metallicity plot as shown in Figure 1. For stars with low $\mathrm{S} / \mathrm{N}$ spectra and no available EW measurements, we check with the best-fit RV template on the velocity measurements to assess whether the stars are metal-poor or not. We assess the members and nonmembers in the Tuc III stream subjectively in this section. In Section 3.4, we discuss the membership probability of all the observed stars using an objective Bayesian approach.

Among the 31 member stars in the Tuc III stream, 29 were observed by AAT, and 20 of them are the first identified members in the tidal tails. Two are BHB members (DES J234654.06 -594331.7 and DES J235248.09-602054.9), one is an RHB 
Table 1

Velocity and Metallicity Measurements of the Observed Stars

\begin{tabular}{|c|c|c|c|c|c|c|c|c|c|c|c|c|c|}
\hline $\mathrm{ID}^{\mathrm{a}}$ & $\begin{array}{l}\alpha_{2000} \\
(\mathrm{deg})\end{array}$ & $\begin{array}{l}\delta_{2000} \\
(\mathrm{deg})\end{array}$ & $\begin{array}{c}g^{\mathrm{b}} \\
(\mathrm{mag})\end{array}$ & $\begin{array}{c}r^{\mathrm{b}} \\
(\mathrm{mag})\end{array}$ & Cat. $^{\text {b }}$ & Inst. $^{\mathrm{c}}$ & $\mathrm{S} / \mathrm{N}$ & $\begin{array}{c}v \\
\left(\mathrm{~km} \mathrm{~s}^{-1}\right)\end{array}$ & $\begin{array}{l}\text { EW } \\
(\AA)\end{array}$ & {$[\mathrm{Fe} / \mathrm{H}]$} & Mem. $^{\text {d }}$ & Prob..$^{\mathrm{e}}$ & Comment \\
\hline \multicolumn{14}{|c|}{ Tuc III Leading/West Tail } \\
\hline DES J234319.89-592540.8 & 355.83288 & -59.42799 & 18.194 & 17.719 & DR1 & AAT & 22.2 & $-72.93 \pm 0.98$ & $5.01 \pm 0.36$ & $\ldots$ & 0 & 0.00 & \\
\hline DES J234327.66-593542.1 & 355.86526 & -59.59502 & 18.931 & 18.465 & DR1 & AAT & 12.9 & $-116.19 \pm 1.79$ & $1.30 \pm 0.32$ & $-2.97 \pm 0.29$ & 1 & 1.00 & \\
\hline DES J234350.83-593925.6 & 355.96177 & -59.65712 & 17.678 & 17.151 & Y2Q & AAT & 33.8 & $-115.46 \pm 0.76$ & $1.83 \pm 0.30$ & $-2.83 \pm 0.18$ & 1 & 1.00 & Binary \\
\hline DES J234434.87-594407.7 & 356.14531 & -59.73549 & 18.339 & 17.835 & DR1 & AAT & 20.8 & $-115.27 \pm 1.23$ & $2.15 \pm 0.49$ & $-2.51 \pm 0.27$ & 1 & 0.99 & \\
\hline DES J234437.03-595405.6 & 356.15431 & -59.90157 & 17.005 & 16.391 & DR1 & AAT & 40.7 & $-75.88 \pm 0.77$ & $5.49 \pm 0.27$ & $\cdots$ & 0 & 0.00 & \\
\hline DES J234521.78-593131.3 & 356.34076 & -59.52536 & 19.340 & 18.883 & DR1 & AAT & 9.6 & $-116.09 \pm 2.43$ & $1.65 \pm 0.52$ & $-2.62 \pm 0.36$ & 1 & 0.99 & \\
\hline DES J234531.06-593908.6 & 356.37941 & -59.65240 & 17.672 & 17.127 & DR1 & AAT & 36.0 & $-110.38 \pm 0.84$ & $2.27 \pm 0.38$ & $-2.59 \pm 0.20$ & 1 & 0.98 & \\
\hline DES J234642.46-601828.1 & 356.67692 & -60.30780 & 19.046 & 18.576 & DR1 & AAT & 9.8 & $-114.02 \pm 3.39$ & $2.01 \pm 0.49$ & $-2.44 \pm 0.34$ & 1 & 0.98 & \\
\hline DES J234642.83-593152.8 & 356.67844 & -59.53133 & 19.487 & 19.038 & DR1 & AAT & 8.7 & $-114.70 \pm 3.08$ & $1.85 \pm 0.79$ & $-2.47 \pm 0.52$ & 1 & 0.97 & \\
\hline DES J234654.06-594331.7 & 356.72524 & -59.72548 & 17.240 & 17.395 & DR1 & AAT & 21.7 & $-109.61 \pm 1.61$ & $\ldots$ & $\ldots$ & 1 & 0.91 & BHB \\
\hline DES J234702.14-594843.2 & 356.75890 & -59.81200 & 18.459 & 17.967 & DR1 & AAT & 17.7 & $-109.26 \pm 1.52$ & $1.99 \pm 0.59$ & $-2.58 \pm 0.34$ & 1 & 0.99 & \\
\hline DES J234719.86-595348.0 & 356.83277 & -59.89668 & 19.449 & 18.989 & DR1 & AAT & 8.9 & $-110.65 \pm 2.68$ & $\ldots$ & $\ldots$ & 1 & 0.92 & \\
\hline DES J234727.52-591504.9 & 356.86466 & -59.25137 & 18.296 & 17.789 & DR1 & AAT & 23.1 & $-111.07 \pm 0.99$ & $2.23 \pm 0.43$ & $-2.48 \pm 0.24$ & 1 & 1.00 & \\
\hline DES J234949.16-602020.5 & 357.45482 & -60.33902 & 18.137 & 17.596 & DR1 & AAT & 6.2 & $-113.59 \pm 3.12$ & $\ldots$ & $\ldots$ & 0 & 0.74 & \\
\hline DES J235158.27-591210.9 & 357.99278 & -59.20303 & 17.541 & 17.271 & Y2Q & AAT & 31.7 & $-88.46 \pm 0.84$ & $4.98 \pm 0.28$ & $\ldots$ & 0 & 0.00 & \\
\hline DES J235248.09-602054.9 & 358.20036 & -60.34857 & 17.265 & 17.404 & DR1 & AAT & 16.3 & $-105.58 \pm 2.57$ & $\ldots$ & $\ldots$ & 1 & 0.90 & BHB \\
\hline \multirow[t]{2}{*}{ DES J235349.12-593245.4 } & 358.45467 & -59.54593 & 18.524 & 18.025 & DR1 & AAT & 17.1 & $-102.56 \pm 2.51$ & $2.41 \pm 0.46$ & $-2.34 \pm 0.25$ & 1 & 0.98 & \\
\hline & & & & & & IMACS & 28.8 & $-103.61 \pm 1.04$ & $2.60 \pm 0.21$ & $-2.25 \pm 0.12$ & & $\cdots$ & \\
\hline DES J235425.88-594103.3 & 358.60784 & -59.68424 & 20.288 & 20.008 & DR1 & IMACS & 6.8 & $-102.65 \pm 2.64$ & $\ldots$ & $\ldots$ & 1 & $\ldots$ & Subgiant \\
\hline DES J235435.00-593946.0 & 358.64582 & -59.66279 & 20.695 & 20.493 & DR1 & IMACS & 4.1 & $-100.63 \pm 3.24$ & $\cdots$ & $\cdots$ & 1 & $\cdots$ & MSTO \\
\hline DES J235439.51-594118.7 & 358.66463 & -59.68854 & 19.997 & 19.611 & DR1 & IMACS & 9.7 & $-71.48 \pm 1.75$ & $1.87 \pm 0.29$ & $\cdots$ & 0 & $\cdots$ & \\
\hline \multicolumn{14}{|c|}{ Tuc III Core ${ }^{f}$} \\
\hline DES J235532.68-593115.0 & 358.88616 & -59.52083 & 16.090 & 15.344 & DR1 & AAT & 125.7 & $-102.89 \pm 0.51$ & $3.75 \pm 0.22$ & $-2.28 \pm 0.10$ & 1 & 0.99 & \\
\hline DES J235549.92-593259.7 & 358.95799 & -59.54990 & 17.400 & 16.815 & DR1 & AAT & 22.0 & $-101.94 \pm 1.40$ & $2.84 \pm 0.29$ & $-2.37 \pm 0.14$ & 1 & 1.00 & \\
\hline DES J235620.76-593310.2 & 359.08651 & -59.55282 & 18.857 & 18.374 & DR1 & AAT & 11.6 & $-101.83 \pm 2.19$ & $2.33 \pm 0.27$ & $-2.31 \pm 0.16$ & 1 & 0.99 & \\
\hline DES J235650.50-593421.0 & 359.21042 & -59.57250 & 19.950 & 19.496 & DR1 & AAT & 6.0 & $-102.12 \pm 4.99$ & $\ldots$ & $\ldots$ & 1 & 0.83 & \\
\hline DES J235707.46-593743.0 & 359.28110 & -59.62861 & 19.727 & 19.296 & DR1 & AAT & 5.7 & $-104.53 \pm 4.82$ & $\ldots$ & $\ldots$ & 1 & 0.81 & \\
\hline DES J235726.04-593938.2 & 359.35851 & -59.66061 & 19.265 & 18.800 & DR1 & AAT & 13.2 & $-101.89 \pm 2.64$ & $\ldots$ & $\ldots$ & 1 & 0.89 & \\
\hline DES J235730.25-592930.7 & 359.37602 & -59.49186 & 19.342 & 18.878 & DR1 & AAT & 8.1 & $-100.06 \pm 3.19$ & $\ldots$ & $\ldots$ & 1 & 0.88 & \\
\hline DES J235738.50-593611.7 & 359.41040 & -59.60325 & 17.173 & 16.569 & DR1 & AAT & 45.9 & $-99.58 \pm 0.71$ & $2.66 \pm 0.25$ & $-2.50 \pm 0.13$ & 1 & 1.00 & \\
\hline DES J235745.46-593726.5 & 359.43941 & -59.62401 & 19.806 & 19.379 & DR1 & AAT & 8.2 & $-100.01 \pm 3.29$ & $\ldots$ & $\ldots$ & 1 & 0.88 & \\
\hline \multicolumn{14}{|c|}{ Tuc III Trailing/East Tail } \\
\hline DES J235853.63-595952.2 & 359.72345 & -59.99784 & 18.956 & 18.467 & DR1 & AAT & 10.3 & $-121.84 \pm 2.14$ & $3.87 \pm 0.63$ & $\ldots$ & 0 & 0.00 & \\
\hline \multirow[t]{2}{*}{ DES J235855.20-591242.4 } & 359.73001 & -59.21177 & 18.701 & 18.219 & DR1 & AAT & 9.8 & $-78.54 \pm 2.86$ & $2.16 \pm 0.29$ & $\cdots$ & 0 & 0.00 & \\
\hline & & & & & & IMACS & 6.6 & $-74.02 \pm 1.52$ & $\cdots$ & $\cdots$ & & $\cdots$ & \\
\hline DES J000104.89-594814.4 & 0.27039 & -59.80400 & 18.833 & 18.394 & DR1 & AAT & 8.0 & $-85.13 \pm 4.37$ & $2.19 \pm 0.60$ & $\ldots$ & 0 & 0.49 & \\
\hline DES J000234.74-593056.8 & 0.64473 & -59.51578 & 19.341 & 18.881 & DR1 & AAT & 5.7 & $-92.26 \pm 4.46$ & $\cdots$ & $\cdots$ & 1 & 0.78 & \\
\hline DES J000344.78-600048.2 & 0.93656 & -60.01339 & 19.109 & 18.670 & DR1 & AAT & 7.8 & $-129.28 \pm 3.42$ & $\cdots$ & $\cdots$ & 0 & 0.00 & \\
\hline DES J000347.26-593114.6 & 0.94693 & -59.52072 & 19.104 & 18.646 & DR1 & AAT & 7.1 & $-93.82 \pm 3.38$ & $\cdots$ & $\cdots$ & 1 & 0.86 & \\
\hline DES J000427.21-593648.3 & 1.11336 & -59.61342 & 18.771 & 18.283 & DR1 & AAT & 10.7 & $-94.58 \pm 2.58$ & $\cdots$ & $\cdots$ & 1 & 0.87 & \\
\hline DES J000529.05-600323.4 & 1.37102 & -60.05651 & 17.543 & 17.318 & DR1 & AAT & 21.6 & $-91.05 \pm 2.97$ & $\ldots$ & $\ldots$ & 1 & 0.85 & RHB \\
\hline
\end{tabular}




\begin{tabular}{|c|c|c|c|c|c|c|c|c|c|c|c|c|c|}
\hline $\mathrm{ID}^{\mathrm{a}}$ & $\begin{array}{l}\alpha_{2000} \\
(\mathrm{deg})\end{array}$ & $\begin{array}{l}\delta_{2000} \\
(\mathrm{deg})\end{array}$ & $\begin{array}{c}g^{\mathrm{b}} \\
(\mathrm{mag})\end{array}$ & $\begin{array}{c}r^{\mathrm{b}} \\
(\mathrm{mag})\end{array}$ & Cat. $^{\mathrm{b}}$ & Inst. $^{c}$ & $\mathrm{~S} / \mathrm{N}$ & $\begin{array}{c}v \\
\left(\mathrm{~km} \mathrm{~s}^{-1}\right)\end{array}$ & $\begin{array}{l}\text { EW } \\
(\mathrm{A})\end{array}$ & {$[\mathrm{Fe} / \mathrm{H}]$} & Mem. $^{\mathrm{d}}$ & Prob. $^{\mathrm{e}}$ & Comment \\
\hline DES J000548.69-593406.1 & 1.45288 & -59.56835 & 16.770 & 16.123 & $\mathrm{Y} 2 \mathrm{Q}$ & AAT & 40.4 & $-92.01 \pm 0.70$ & $2.34 \pm 0.35$ & $-2.75 \pm 0.18$ & 1 & 1.00 & \\
\hline DES J000639.63-591658.5 & 1.66513 & -59.28291 & 18.813 & 18.316 & DR1 & AAT & 9.8 & $-92.11 \pm 2.30$ & $2.09 \pm 0.27$ & $-2.46 \pm 0.16$ & 1 & 0.99 & \\
\hline DES J000727.58-593357.0 & 1.86493 & -59.56584 & 19.321 & 18.865 & Y2Q & AAT & 7.3 & $-92.58 \pm 3.35$ & $\cdots$ & $\cdots$ & 1 & 0.82 & \\
\hline DES J000758.93-594729.2 & 1.99552 & -59.79145 & 18.530 & 18.038 & DR1 & AAT & 11.7 & $-99.87 \pm 2.08$ & $5.83 \pm 0.58$ & $\ldots$ & 0 & 0.00 & \\
\hline
\end{tabular}

Note. Only stars with $-140 \mathrm{~km} \mathrm{~s}^{-1}<v_{\text {hel }}<-70 \mathrm{~km} \mathrm{~s}^{-1}$ are presented here.

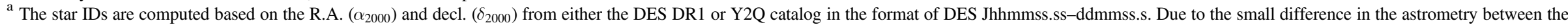

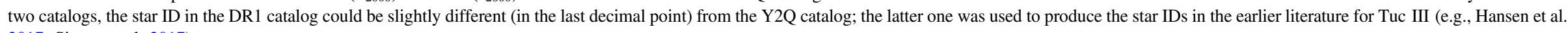
2017; Simon et al. 2017).

${ }^{\mathrm{b}}$ Quoted magnitudes represent the dereddened PSF magnitude in either the DR1 or Y2Q catalog.

${ }^{\mathrm{c}}$ Telescopes and instruments used for obtaining the spectra of the targets.

$\mathrm{d}$ The membership of the Tuc III stream stars through a subjective membership identification. (See details in Section 3.1.) Mem $=1$ are members; Mem $=0$ are nonmembers.

e The membership probability of Tuc III stream stars through an objective mixture model. Measurements from the AAT observations are used for modeling. (See details in Section 3.4.)

${ }^{\mathrm{f}}$ These nine Tuc III core members are also observed by Simon et al. (2017).

(This table is available in its entirety in machine-readable form.) 
member (DES J000529.05-600323.4), and the remaining stars are on the RGB. The RHB star shows no velocity variation from multiple measurements over several nights of AAT observations in 2016, so we conclude that it is not an RR Lyrae star. We note that RHB stars are not common for dwarf galaxies at this luminosity and age, but from the velocity, it is consistent with the Tuc III member stars. Including or excluding this star does not change the kinematic properties of the stream.

In order to check for possible binary motions of the stream member stars, we combined the spectra from the 2016 and 2017 AAT runs separately to provide independent measurements with a time baseline of $\sim 13$ months for each star. We note that only the member stars that are observed in both AAT-Field-1 and AAT-Field-3 have repeated measurements after 13 months (see Figure 1). We find that RGB member DES J234350.83 -593925.6 is likely a binary, because we measured an RV of $v_{\text {hel }}=-122.2 \pm 0.8 \mathrm{~km} \mathrm{~s}^{-1}$ during the 2016 run (average $\mathrm{MJD}=57,589) \quad$ and $\quad v_{\text {hel }}=-99.4 \pm 1.4 \mathrm{~km} \mathrm{~s}^{-1}$ on 2017 August 22 (MJD = 57,987). Its measured velocity from the combined spectra $\left(v_{\text {hel }}=-115.5 \pm 0.8 \mathrm{~km} \mathrm{~s}^{-1}\right)$ is very similar to that of other stream members nearby; furthermore, it also has a very low metallicity $([\mathrm{Fe} / \mathrm{H}]=-2.8 \pm 0.2)$. We therefore conclude that it is a stream member. However, we exclude this star from the kinematic analysis in later sections. All remaining AAT members do not show large velocity variations with the data we have obtained.

In contrast to the AAT, IMACS probes deeper but with a much smaller field of view. One subgiant (DES J235425.88 -594103.3) and one MSTO star (DES J235435.00-593946.0) were uniquely detected by IMACS in one of the masks. In addition, RGB member DES J235349.12-593245.4 was observed by both AAT and IMACS and shows no velocity difference between the two.

We then discuss the nonmembers that have velocities close to the systemic velocity of the stream. Specifically, we list all of the nonmember stars that are in the velocity range of -140 and $-70 \mathrm{~km} \mathrm{~s}^{-1}$.

1. A few candidate stars, including DES J234319.89 -592540.8, DES J234437.03-595405.6, DES J235853.63 -595952.2, DES J000344.78-600048.2, and DES J000 758.93-594729.2, have relatively large CaT EWs, suggesting that they are nonmembers.

2. DES J234949.16-602020.5 has a very low $\mathrm{S} / \mathrm{N}$, so no $\mathrm{EW}$ is measured, but a metal-rich RV template was selected as the best-fit template. ${ }^{48}$

3. DES J235855.20-591242.4 has a small CaT EW, but its velocity is more than $20 \mathrm{~km} \mathrm{~s}^{-1}$ off from the bulk velocity of the stream at its location. The independent RV measurements from AAT and IMACS are consistent within the $1 \sigma$ uncertainty, suggesting that it is not in a binary system.

4. DES J235158.27-591210.9 is similar. It also has a small EW but a large velocity offset $\left(>20 \mathrm{~km} \mathrm{~s}^{-1}\right)$ from the member stars at a similar location on the sky.

5. DES J000104.89-594814.4 has a small EW, and its velocity is about $10 \mathrm{~km} \mathrm{~s}^{-1}$ off from the bulk velocity of the stream at the location. Furthermore, its position on the color-magnitude diagram (CMD) is slightly offset from the other members.

\footnotetext{
48 The membership of this star will be discussed further in Section 3.4
}

We suggest that for the last two nonmembers mentioned above, i.e., DES J235158.27-591210.9 and DES J000104.89 -594814.4 , more observations would be useful to check the possibility that they could be stream members with velocities offset from the stream by binary orbital motions.

In order to derive the kinematic properties of the stream in the following sections, we transformed the member stars from celestial equatorial coordinates $(\alpha, \delta)$ to stream coordinates $(\Lambda, B)$ using Euler angles $\left(\phi, \theta, \psi=264^{\circ} .23,120^{\circ} .29,267^{\circ} .51\right)$, where $\phi, \theta$ are derived from the pole of the Tuc III stream from Shipp et al. (2018) by assuming a great circle orbit on the sky and $\psi$ is chosen so that the center of the Tuc III core has $\Lambda=0$. We also list the transformation matrix in Appendix A. The member stars in the stream coordinates $(\Lambda, B)$ are shown in the top panel of Figure 2.

We observed candidate stars that are roughly \pm 0.7 from the Tuc III stream track, taking into account the stream width of $\sigma_{w}=0^{\circ} .13$ (or FWHM $=0.3$ ) from Drlica-Wagner et al. (2015) at the discovery of the stream. The reanalysis from Shipp et al. (2018) with improved data sets indicates the stream is slightly wider at $\sigma_{w}=0$. 18 . Surprisingly, we found three apparent member stars at least $3 \sigma$ away from $B=0$ (see Figure 2), including one BHB member $\left(B=-0^{\circ} .69\right)$, one RGB member $(B=-0.692)$, and one RHB member $(B=-0.54)$. Finding three out of 22 members at $B>3 \sigma_{w}$ may indicate that the stream profile is non-Gaussian. This can naturally arise if Tuc III is a globular cluster since, while $~ 5 \%$ of stars will escape per relaxation time due to tidal stripping (Hénon 1961), $\sim 1 \%$ will escape from the core (e.g., Spitzer 1987; Baumgardt et al. 2002; Alexander \& Gieles 2012) with a much larger velocity dispersion due to multibody interactions. The $\sim 17 \%$ of stars ejected from the core would thus produce a much broader stream than the stars that were tidally stripped. Of course, this argument will not apply if Tuc III is a dwarf galaxy.

A non-Gaussian profile across the stream can also arise due to epicyclic motion along the stream (Küpper et al. 2008, 2010, 2012). In particular, away from pericenter, the epicycles bunch up and can almost overlap (see, e.g., Figure 9 of Küpper et al. 2012). This can create streams with stars significantly off the main track.

Alternatively, this may imply that one or some of these three stars are not true members of the Tuc III stream, especially for the RHB member. As we noted earlier, since the RHB star does not seem to be an RR Lyrae star, it is very uncommon for dwarf galaxies at this luminosity to have an RHB member. Proper motion from Gaia Data Release 2 (DR2) ${ }^{49}$ can further assess the membership of these stars.

Shipp et al. (2018) estimated that the stellar mass of the stream is about $3.8 \times 10^{3} M_{\odot}$. Assuming a Chabrier (2001) initial mass function with an age of $12.5 \mathrm{Gyr}$ and metallicity of $[\mathrm{Fe} / \mathrm{H}]=-2.3$, we estimate a total of $\sim 30 \pm 5$ member stars brighter than $g \sim 19.5$ in the stream from 100 realizations of dwarf galaxy stellar populations randomly sampled using ugali. ${ }^{50}$ As a comparison, among all the confirmed members from the tails and the core, 26 members have $g<19.5$. For most of the AAT spectra, we get $\mathrm{S} / \mathrm{N} \sim 7$ at $g \sim 19.5$; except for stars that were uniquely observed in the field of AATField3, where only a $2 \mathrm{hr}$ exposure was taken, we get $\mathrm{S} /$ $\mathrm{N} \sim 4$ at $g \sim 19.5$. About $90 \%$ of the target stars that are

\footnotetext{
49 See https://www.cosmos.esa.int/web/gaia/dr2 for more details.

${ }^{50}$ https://github.com/DarkEnergySurvey/ugali
} 

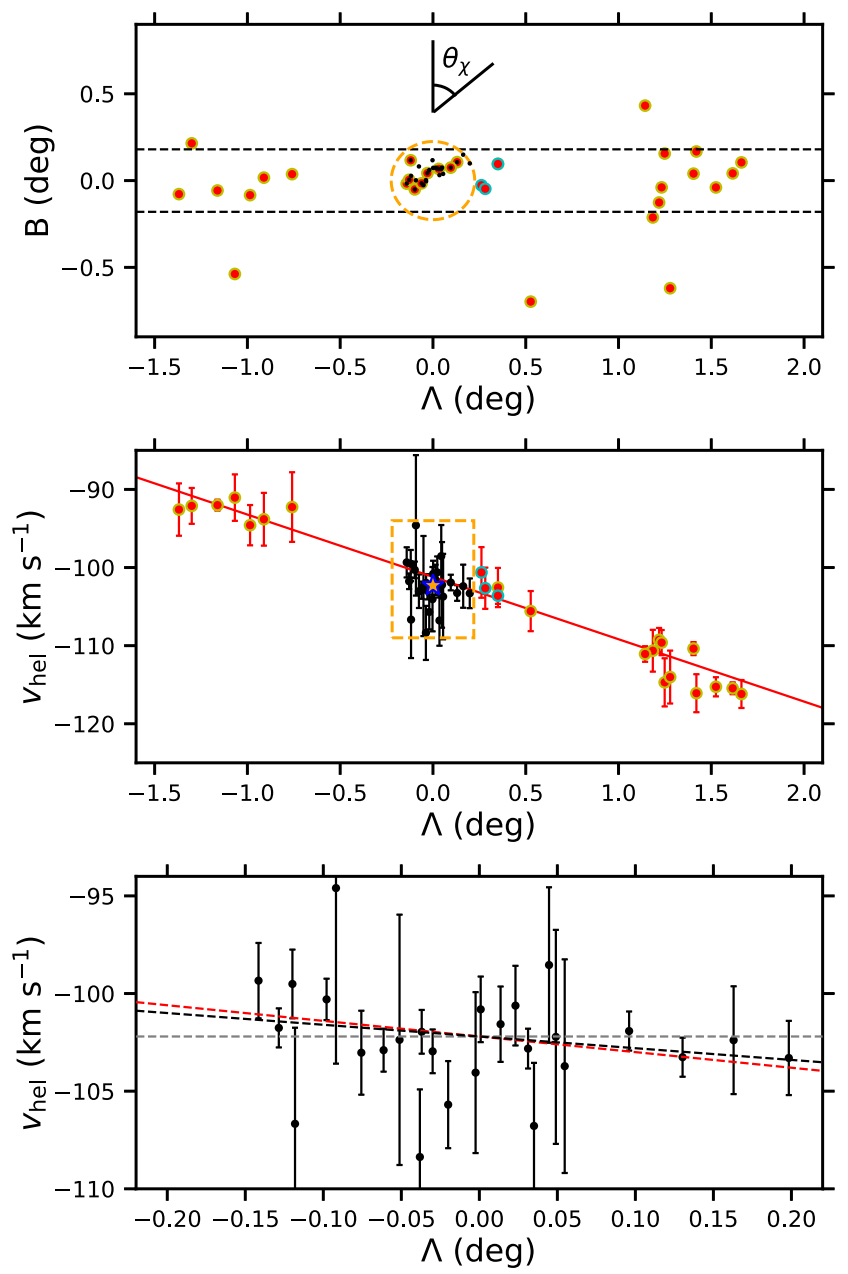

Figure 2. Top: members confirmed in this work in the Tuc III stream coordinates $(\Lambda, \mathrm{B})$, where the center of the Tuc III core is at $(\Lambda, B)=(0,0)$. Symbols are the same as in Figure 1. Also plotted in black dots are the member stars of the Tuc III core from Simon et al. (2017), nine of which overlap with the AAT confirmed members. The black dashed lines show the width $\sigma_{w}=0.18(1 \sigma)$ of the stream from Shipp et al. (2018). The orange dashed circle shows the definition of members in Tuc III core $\left(r<2.2 r_{h}\right)$, where all the members confirmed in Simon et al. (2017) are encircled. The definition of position angle $\theta_{\chi}$ is also illustrated. Middle: heliocentric velocity $v_{\text {hel }}$ as a function of stream longitude $\Lambda$ for 22 members in the tidal tails. The RGB member DES J235349.12-593245.4 was observed by both AAT (yellow circles) and IMACS (cyan circles); both measurements are presented. The red line shows the best-fit velocity gradient and systemic velocity from the MCMC fit using the velocities of the tail members only. The black circles represent the velocities of 26 core members from Simon et al. (2017). The orange star shows the systemic velocity of the Tuc III core measured by Simon et al. (2017). The uncertainty is smaller than the size of the symbol. Bottom: zoom-in of the dashed orange rectangle in the middle panel, in which the velocities of the 26 members from Simon et al. (2017) are also presented. The black dashed line shows the best-fit velocity gradient from these 26 core members, the red dashed line indicates the gradient from the tails (i.e., same as the red solid line in the middle panel), and the gray dashed line indicates a no-gradient model. The gradient derived from core members alone $\left(-6.0 \pm 3.9 \mathrm{~km} \mathrm{~s}^{-1} \mathrm{deg}^{-1}\right)$ is similar to what is detected in the stream $\left(-8.0 \pm 0.4 \mathrm{~km} \mathrm{~s}^{-1} \mathrm{deg}^{-1}\right)$ but with a much larger uncertainty. Due to the relatively large velocity uncertainties and small velocity differences observed in the core, the gradient in the core is statistically insignificant (see more discussion in the text).

brighter than $g \sim 19.5$ have successful $\mathrm{RV}$ measurements. Those $10 \%$ unsuccessful measurements are mostly from spectra in AAT-Field3. We conclude that our sample is near complete at $g<19.5$ for AAT-Field1 and AATField2, which covers roughly $3: 6$ in total. However, the actual stream length is about 4.8 ; therefore, we expect several additional brighter members near the ends of the stream that were not observed in this work.

Drlica-Wagner et al. (2015) found that the stellar mass of the Tuc III dwarf galaxy candidate is around $800 M_{\odot}$, which is about $21 \%$ of the total stellar mass of the stream. Assuming a Plummer profile, the enclosed stellar mass within two half-light radii $\left(2 r_{\mathrm{h}}\right)$, close to the definition of the Tuc III core here, is about $80 \%$ of the total mass of the dwarf galaxy, i.e., $17 \%$ of the total stellar mass of the stream. As a comparison, for all the members at $g<19.5$, we identified six members in the core and 20 members in the tails, which confirms that the member stars in the core account for $6 / 26 \sim 23 \%$ of the total members in the stream. As we expect additional brighter members would be found near the ends of the stream as discussed above, this ratio would become lower after finding more tail members.

\subsection{Velocity Gradient}

As shown in Figure 1, a clear velocity gradient with increasing velocity toward larger R.A. $\left(\alpha_{2000}\right.$, or equatorial east) is present in the Tuc III stream. In this section, we calculate the systemic velocity and velocity gradient using the tail stars measured in this paper and compare the systemic velocity with what was derived in Simon et al. (2017). We also perform the fit with a different setup to check if the results change with different fitting parameters and data sets. The results are summarized in Table 2.

We first fit the RVs $(v)$ and RV uncertainties $\left(\delta_{v}\right)$ of 21 tail members (after excluding one probable binary member) via a maximum-likelihood approach and a three-parameter likelihood function similar to $\mathrm{Li}$ et al. (2017) to derive the systemic velocity $v_{\text {hel }}$ of the stream, i.e., the system velocity at the center of the Tuc III core $(\Lambda=0)$, the velocity gradient along stream longitude $d v / d \Lambda$, and the velocity dispersion $\sigma_{v}$ :

$$
\log \mathcal{L}=-\frac{1}{2}\left[\sum_{i=1}^{N} \log \left(\sigma_{v}^{2}+\delta_{v_{i}}^{2}\right)+\sum_{n=1}^{N} \frac{\left(v_{i}-v_{\text {hel }}-\frac{d v}{d \Lambda} \Lambda_{i}\right)^{2}}{\delta_{v_{i}}^{2}+\sigma_{v}^{2}}\right]
$$

Since Simon et al. (2017) used flat priors to fit the systemic velocity and velocity dispersion, we use flat priors for all three parameters to have a direct comparison with Simon et al. (2017) later. $^{51}$ The posterior distribution from the MCMC sampler is shown in Figure 3, and the best-fit values are

$$
\begin{aligned}
v_{\text {hel } \mid(\Lambda=0)} & =-101.2 \pm 0.5 \mathrm{~km} \mathrm{~s}^{-1} \\
d v_{\text {hel }} / d \Lambda & =-8.0 \pm 0.4 \mathrm{~km} \mathrm{~s}^{-1} \mathrm{deg}^{-1}, \\
\sigma_{v} & =0.9_{-0.5}^{+0.6} \mathrm{~km} \mathrm{~s}^{-1}
\end{aligned}
$$

where we report the 50th percentile of the posterior and the uncertainty is calculated from the 16th and 84th percentiles. At a distance of $25 \mathrm{kpc}$, the velocity gradient of $d v_{\text {hel }} / d \Lambda=$ $-8.0 \pm 0.4 \mathrm{~km} \mathrm{~s}^{-1} \mathrm{deg}^{-1}$ corresponds to $18.3 \mathrm{~km} \mathrm{~s}^{-1} \mathrm{kpc}^{-1}$ projected on the sky in the heliocentric frame. The best-fit velocity gradient and systemic velocity, along with the velocities of the tail members, are shown in the middle panel of Figure 2.

\footnotetext{
51 The overall posterior distribution will be smaller for the velocity dispersion if the Jeffreys prior is used instead (see, e.g., Kim et al. 2015; Li et al. 2018)
} 
Table 2

Best Fit on Kinematics with Different Data Sets or Fitting Parameters

\begin{tabular}{|c|c|c|c|c|c|}
\hline Fitting & No. of Stars & $\begin{array}{c}v_{\text {hel }} \\
\left(\mathrm{km} \mathrm{s}^{-1}\right)\end{array}$ & $\begin{array}{c}d v / d \Lambda \text { or } d v / d \chi \\
\left(\mathrm{km} \mathrm{s}^{-1} \mathrm{deg}^{-1}\right)\end{array}$ & $\begin{array}{c}\sigma_{v} \\
\left(\mathrm{~km} \mathrm{~s}^{-1}\right)\end{array}$ & $\begin{array}{c}\theta_{\chi} \\
(\mathrm{deg})\end{array}$ \\
\hline 3-parameter, tails only (default) & 21 & $-101.2 \pm 0.5$ & $-8.0 \pm 0.4$ & $0.9_{-0.5}^{+0.6}$ & $\cdots$ \\
\hline 4-parameter, tails only & 21 & $-101.2 \pm 0.5$ & $-8.2_{-0.6}^{+0.4}$ & $0.9_{-0.5}^{+0.6}$ & $81 \pm 14$ \\
\hline 3-parameter, tails + core & 30 & $-101.3 \pm 0.3$ & $-7.9 \pm 0.4$ & $0.6 \pm 0.4$ & $\ldots$ \\
\hline 4-parameter, tails+core & 30 & $-101.3 \pm 0.3$ & $-8.1_{-0.5}^{+0.4}$ & $0.6 \pm 0.4$ & $80 \pm 12$ \\
\hline 3-parameter, core (Simon et al. 2017) & 26 & $-102.2 \pm 0.4$ & $-6.0 \pm 3.9$ & $<1.3$ & $\cdots$ \\
\hline 4-parameter, core (Simon et al. 2017) & 26 & $-102.2 \pm 0.4$ & $-6.7 \pm 6.1$ & $<1.3$ & $103_{-68}^{+43}$ \\
\hline 11-parameter membership, tail+core & 552 & $-101.4 \pm 0.5$ & $-8.4_{-0.5}^{+0.4}$ & $0.8 \pm 0.4$ & $86 \pm 13$ \\
\hline
\end{tabular}

Note. All values reported here (and in this paper) are from the 50th percentile of the posterior probability distributions. The uncertainties are from the 16th and 84th percentiles of the posterior probability distributions. For the upper limit, the $95 \%$ confidence level is used.

We note that this is a fit using only the 21 out of 22 members in the tails (one is excluded because of apparent binarity). We did not use the other nine member stars in the core that were both measured in this work, as well as in Simon et al. (2017). Therefore, the fit gives an independent check on the systemic velocity of the Tuc III core. The systemic velocity of the Tuc III core is $-102.3 \pm 0.4 \mathrm{~km} \mathrm{~s}^{-1}$ (Simon et al. 2017) from a sample of 26 core members. The difference between the two, $\sim 1 \mathrm{~km} \mathrm{~s}^{-1}$, is about $1.5 \sigma$ of the joint uncertainty. To test the origin of this slight velocity difference, we compare the individual member stars observed with both AAT and IMACS (mostly from Simon et al. 2017), as shown in Figure 4. All stars have consistent velocity measurements within a $1 \sigma$ uncertainty except for DES J235738.50-593611.7, for which the difference is $\sim 2 \sigma$. If we use only the nine core members that are measured in both works, we get $v_{\text {hel }}=-101.6 \pm 0.5 \mathrm{~km} \mathrm{~s}^{-1}$ from AAT measurements and $v_{\text {hel }}=-102.0 \pm 0.4 \mathrm{~km} \mathrm{~s}^{-1}$ from Simon et al. (2017). We conclude that the systematic offset between the two instruments is minimal. This is consistent with our comparison of the measurements from these two instruments in $\mathrm{Li}$ et al. (2018). This also confirms that these nine core members do not show any binary motions.

We also measured the velocity gradient and dispersion with 21 tail members plus nine core members measured in this work. The results are consistent with fitting the tail sample alone.

We note that we fit the gradient along $\Lambda$ (i.e., $B=0$ ) in the earlier analysis. Similar to Li et al. (2017), we introduce an additional degree of freedom on position angle $\theta_{\chi}$, which is defined to be north-to-east in the stream coordinates (see illustration in the top panel of Figure 2) and run a fourparameter fit in stream coordinates to check the possibility that the velocity gradient $d v / d \chi$ is not aligned with the stream. We found very similar results (see Table 2) to those from the threeparameter fit, and $\theta_{\chi}=81^{\circ} \pm 14^{\circ}$ is consistent with the case where the gradient is aligned with the stream $\left(\theta_{\chi}=90^{\circ}\right)$.

\subsubsection{Equilibrium in the Tuc III Core?}

Enlightened by the fact that Tuc III had a very close pericenter passage (see details in discussions in Section 4.1.2, as well as in Erkal et al. 2018), we examine the state of equilibrium in the Tuc III core; specifically, we search for signatures of velocity gradient in the core. We applied the same four-parameter fit to the 26 core members measured in Simon et al. (2017). ${ }^{52}$ We found a velocity gradient of $d v / d \chi=-6.7 \pm 6.1 \mathrm{~km} \mathrm{~s}^{-1} \mathrm{deg}^{-1}$ and a position

\footnotetext{
$\overline{52}$ For stars that have multiple measurements, the one with the highest $\mathrm{S} / \mathrm{N}$ was used.
}

angle of $\theta_{\chi}=103^{\circ+42 \circ}{ }_{-58}^{\circ}$ for the Tuc III core, which is consistent with the gradient in the Tuc III stream. However, the large uncertainties on both the gradient and the position angle indicate that the velocity gradient is poorly detected. If we apply a three-parameter fit instead, the corresponding gradient is similar with a slightly smaller uncertainty, at $d v / d \Lambda=$ $-6.0 \pm 3.9 \mathrm{~km} \mathrm{~s}^{-1} \mathrm{deg}^{-1}$. In the bottom panel of Figure 2, we show the velocities of the 26 core members in stream coordinates along with the best-fit gradient from the three-parameter fit. To assess the significance of the velocity gradient model in the Tuc III core, we compute the (logarithmic) Bayes's factor (lnB) comparing the gradient model with the null model (no gradient). (See Trotta 2008 for a review of the Bayes factor and Bayesian model selection.) We find that $\ln B=-1.6$ for both the threeparameter and four-parameter models. Values $>0(<0)$ favor (disfavor) the gradient model, and values within the ranges $0<1<2.5<5(0>-1>-2.5>-5)$ correspond to insignificant, low, moderate, and significant evidence in favor (disfavor) of the gradient model (based on the Jeffreys scale; see Table 1 of Trotta 2008). With only the core data, the gradient model is disfavored compared to the no-gradient model at low statistical significance. We note that, even if the core exhibits the same gradient as the stream, an $\sim 0^{\circ} .3$ extension in $\Lambda$ in the core will only have $\sim 2 \mathrm{~km} \mathrm{~s}^{-1}$ difference between the two ends, which is similar to the uncertainty in the velocity measurements for individual stars. The Tuc III velocity gradient therefore only becomes statistically significant once a large radial extent is observed. If more precise velocity measurements are obtained for stars in the range of $0.05<|\Lambda|<0.5$ (or more members are found in this range), it may be possible to identify the location of the transition between the remaining progenitor and the tidal tails. The transition radius can be further compared with the tidal radius at the pericenter of Tuc III's orbit.

A comparison of the posterior distributions of the velocity gradient and velocity dispersion of the Tuc III tails using 22 tail members from this work and those of the Tuc III core using 26 core members are shown in the left and middle panels of Figure 5. For both data sets, the four-parameter fit described above is used. In Simon et al. (2017), the velocity dispersion of the Tuc III core was not resolved (i.e., $\sigma<1.5 \mathrm{~km} \mathrm{~s}^{-1}$ at $95 \%$ confidence level). As shown in the middle panel of Figure 5, the velocity dispersion of the tails is likely slightly higher than that of the core, though the posterior distributions of the two largely overlap. The larger velocity dispersion in the tails may be a natural consequence of the ongoing tidal disruption. 


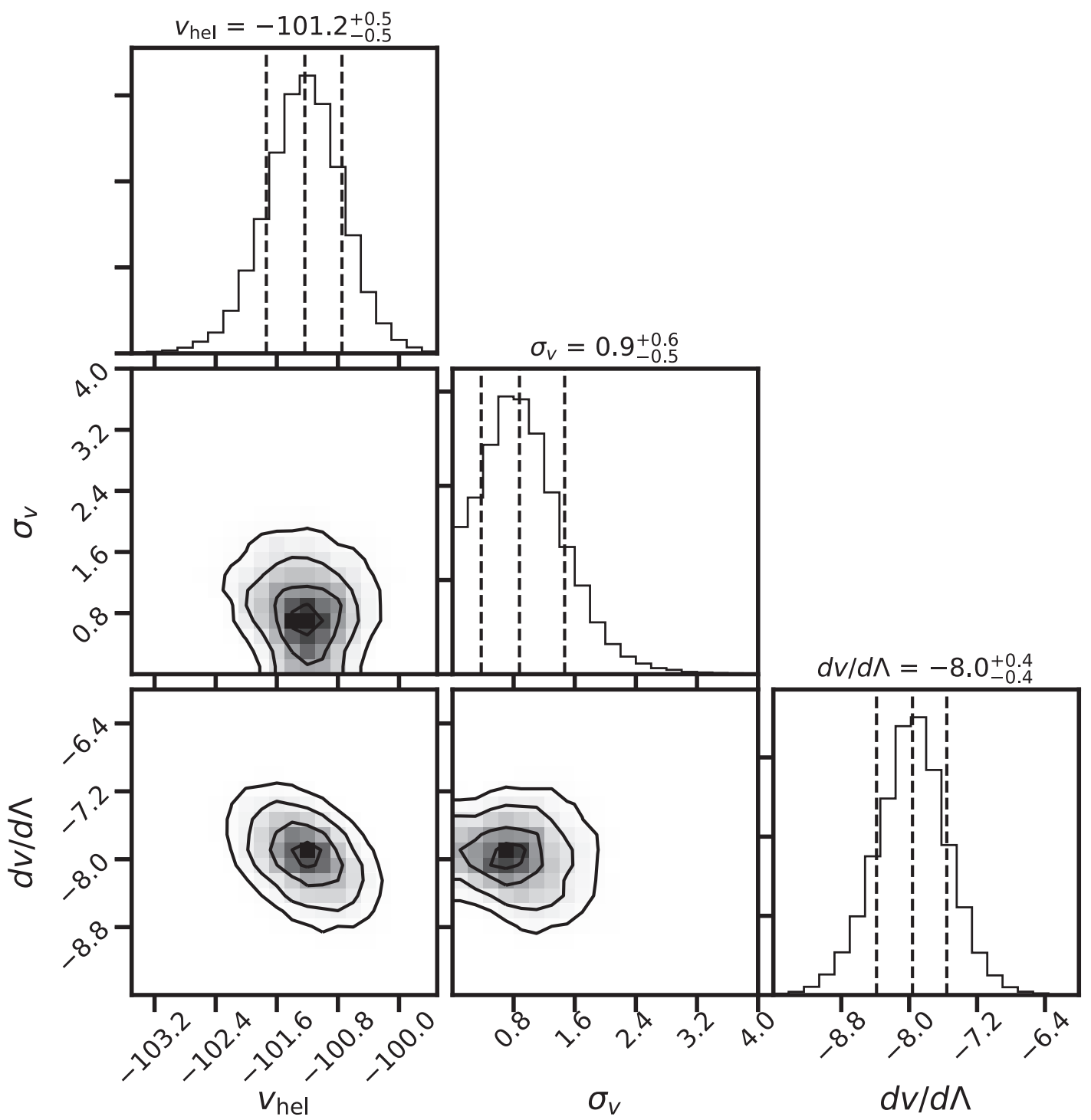

Figure 3. Two-dimensional and marginalized posterior probability distribution from an MCMC sampler using a three-parameter likelihood model. The three parameters are the systemic velocity $v_{\text {hel }}\left(\right.$ in $\mathrm{km} \mathrm{s}^{-1}$ ) at the center of the Tuc III core (i.e., $\Lambda=0$ ), the velocity dispersion $\sigma_{v}$ (in km s $\mathrm{s}^{-1}$ ), and the velocity gradient along stream longitude $d v / d \Lambda$ (in $\mathrm{km} \mathrm{s}^{-1} \mathrm{deg}^{-1}$ ). Dashed lines in the 1D histograms indicate the 16th, 50th, and 84th percentiles of the posterior probability distributions. A large velocity gradient of $-8.0 \pm 0.4 \mathrm{~km} \mathrm{~s}^{-1} \mathrm{deg}^{-1}$ is detected.

\subsection{Metallicity and Metallicity Dispersion}

Among the 31 confirmed member stars in the Tuc III stream, we obtained the metallicity of 16 RGB members, four of which are the brightest RGB members in the Tuc III core and are also measured by Simon et al. (2017) with IMACS. One tail member was measured by both AAT and IMACS in this work. A comparison of the AAT and IMACS measurements for these five members is presented in Figure 4 and shows that there is no systematic offset between the two. The brightest core member (DES J235532) was also observed by Hansen et al. (2017) with high-resolution spectroscopy, and the measured metallicity $([\mathrm{Fe} / \mathrm{H}]=-2.25 \pm 0.18)$ is comparable to what is measured in this work $([\mathrm{Fe} / \mathrm{H}]=-2.28 \pm 0.10)$. We note that although more RGB members in the Tuc III core have metallicity measurements in Simon et al. (2017), we decide to only use the members measured from this work for the analysis of metallicity properties so that the limiting magnitude for both core members and tail members is relatively uniform $(g \lesssim 19)$.

The metallicity of the 16 RGB members from this work spans from $[\mathrm{Fe} / \mathrm{H}]=-2.3$ to $[\mathrm{Fe} / \mathrm{H}]=-3.0$, as shown in Table 1. We found a mean metallicity of $\overline{[\mathrm{Fe} / \mathrm{H}]}=-2.49 \pm$ 0.06 and a metallicity dispersion of $\sigma_{[\mathrm{Fe} / \mathrm{H}]}=0.11_{-0.06}^{+0.07}$, with the posterior distribution presented in Figure 6. As a comparison, Simon et al. (2017) measured a mean metallicity of $\overline{[\mathrm{Fe} / \mathrm{H}]}=-2.44_{-0.08}^{+0.07}$ and an upper limit on the metallicity dispersion of $<0.19$ at the $95.5 \%$ confidence level for the Tuc III core. Similar to the velocity dispersion, the metallicity dispersion from the stream (core+tail) is slightly larger than that in the core (see right panel of Figure 5). The increase in the dispersion might be a hint that the progenitor of the Tuc III 

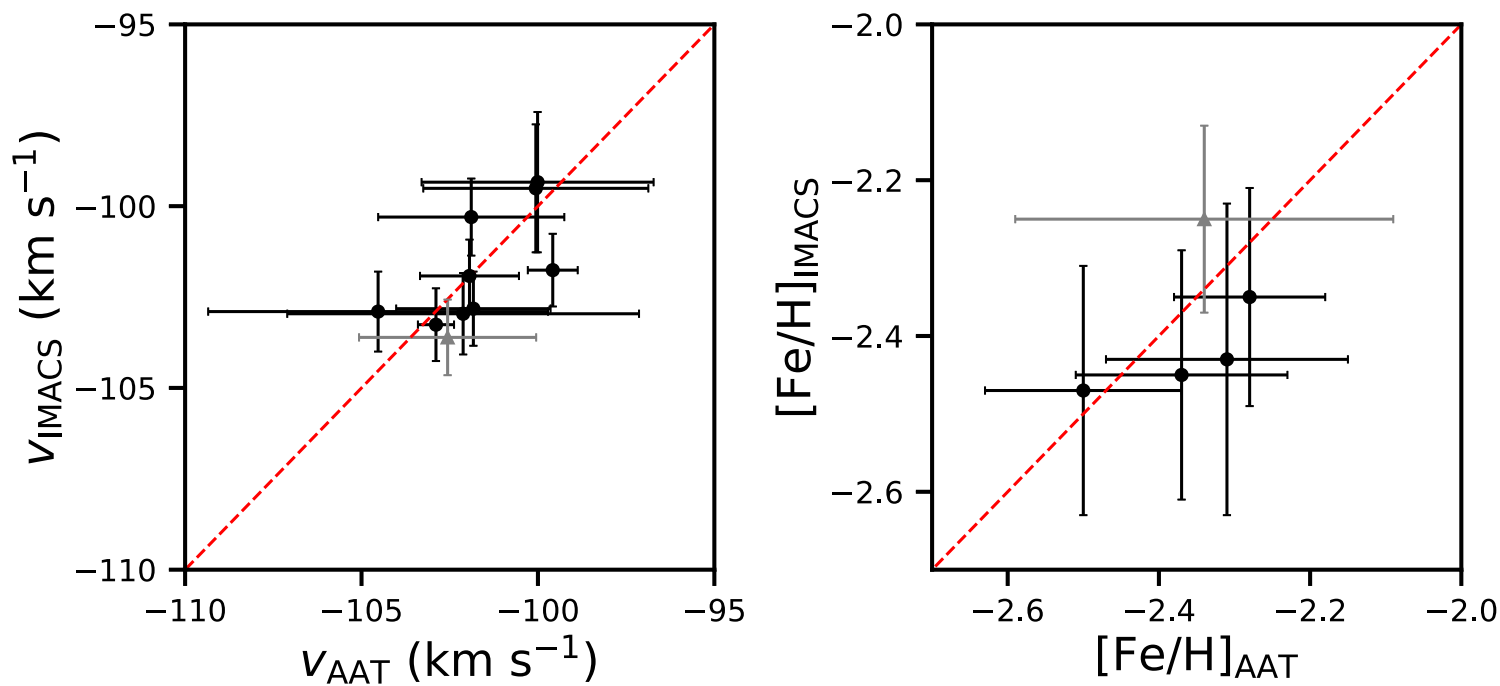

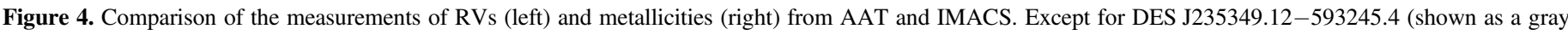

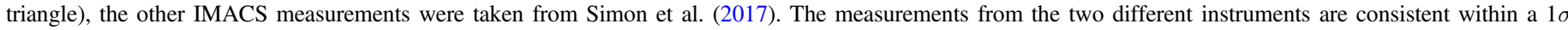

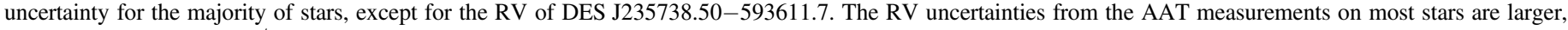
mainly due to the lower $\mathrm{S} / \mathrm{N}$ of the spectra.

stream is more likely to be a dwarf galaxy rather than a star cluster (see discussions in Section 4.1.3). This dispersion is mainly driven by the three most metal-poor RGB members in the stream $([\mathrm{Fe} / \mathrm{H}]<-2.7)$. A comparison of their spectra to those of the core members is displayed in Figure 7. Interestingly, these three most metal-poor RGB members are also among the farthest stream members from the Tuc III center along the stream, as shown in the bottom left panel of Figure 1.

\subsection{Spectroscopic Membership Probability}

We construct a probabilistic mixture model as a cross-check of our membership selection determined in Section 3.1 and to see if the exclusion of an MW component adversely affected our results. We only apply the mixture model to the AAT data to consider a relatively uniform depth across the entire field.

The mixture model likelihood with the Tuc III stream and MW components is written as

$$
P_{\mathrm{Total}}=\left(1-f_{\mathrm{MW}}\right) P_{\mathrm{TucIII}}+f_{\mathrm{MW}} P_{\mathrm{MW}}
$$

where $f_{\mathrm{MW}}$ is the fraction of stars in the MW population. We only use velocity and metallicity data in this mixture model with data vector $\mathcal{D}_{i}=\left(v_{i}, \delta_{v, i},[\mathrm{Fe} / \mathrm{H}]_{i}, \delta_{[\mathrm{Fe} / \mathrm{H}], i}\right)$, where $v_{i}$ and $[\mathrm{Fe} / \mathrm{H}]_{i}$ are the velocity and metallicity of every observed star and $\delta_{v, i}$ and $\delta_{[\mathrm{Fe} / \mathrm{H}], i}$ are the uncertainties of the measurements. Both velocity and metallicity are constructed with Gaussian distributions. We write the Gaussian distribution as

$$
\mathcal{N}\left(v, \sigma^{2}\right)=\frac{1}{\sqrt{2 \pi \sigma^{2}}} \exp \left[-\frac{1}{2} \frac{v^{2}}{\sigma^{2}}\right]
$$

The MW component is

$$
\begin{aligned}
& \mathcal{P}_{\mathrm{MW}}(\mathcal{D})=\mathcal{N}\left(v_{\text {hel }}^{\mathrm{MW}}-v_{i},\left(\sigma_{v}^{\mathrm{MW}}\right)^{2}+\delta_{v, i}^{2}\right) \\
& \times \mathcal{N}\left(\overline{[\overline{\mathrm{Fe}} / \mathrm{H}]_{\mathrm{MW}}}-[\mathrm{Fe} / \mathrm{H}]_{i},\left(\sigma_{[\mathrm{Fe} / \mathrm{H}]}^{\mathrm{MW}}\right)^{2}+\delta_{[\mathrm{Fe} / \mathrm{H}], i}^{2}\right) \text {. }
\end{aligned}
$$

The Tuc III stream model is similar but includes an additional term for the velocity gradient $(d v / d \chi)$ :

$$
\begin{aligned}
\mathcal{P}_{\text {Tuc III }}= & \mathcal{N}\left(v_{\text {hel }}-v_{i}-\frac{d v}{d \chi} \chi_{i}, \sigma_{v}^{2}+\delta_{v, i}^{2}\right) \\
& \times \mathcal{N}\left(\overline{[\mathrm{Fe} / \mathrm{H}]}-[\mathrm{Fe} / \mathrm{H}]_{i}, \sigma_{[\mathrm{Fe} / \mathrm{H}]}^{2}+\delta_{[\mathrm{Fe} / \mathrm{H}], i}^{2}\right) .
\end{aligned}
$$

Overall, we have 11 free parameters: the Tuc III stream $\left(v_{\text {hel }}\right.$, $\left.\sigma_{v}, d v / d \chi, \theta_{\chi}, \overline{[\mathrm{Fe} / \mathrm{H}]}, \sigma_{[\mathrm{Fe} / \mathrm{H}]}\right), \mathrm{MW}\left(v_{\mathrm{hel}}^{\mathrm{MW}}, \sigma_{v}^{\mathrm{MW}}, \overline{[\mathrm{Fe} / \mathrm{H}]}{ }^{\mathrm{MW}}\right.$, $\sigma_{[\mathrm{Fe} / \mathrm{H}]}^{\mathrm{MW}}$ ), and $f_{\mathrm{MW}}$. For the low-S/N stars without metallicity measurements, we average over all possible metallicity values; as the metallicity likelihood is normalized to 1 , this effectively excludes the metallicity term. We assume linear priors for all parameters. We determine the posterior distribution with the MultiNest package (Feroz \& Hobson 2008; Feroz et al. 2009). We compute membership probabilities $(p i)$ by computing the ratio of Tuc III likelihood to the total likelihood $\left(p_{i}=\left(1-f_{\mathrm{MW}}\right) \mathcal{P}_{\text {Tuc III }} / \mathcal{P}_{\text {Total }}\right)$, and we refer to these as the Bayesian membership probabilities (Martinez et al. 2011). The membership is computed for each point in the chain, and the median value is adopted as the final $p i$.

We find 31 stars with nonzero membership $\left(p_{i}>0.001\right)$ and overall membership $\sum p_{i}=28.3$. The 29 members (AAT only) in Section 3.1 all have $p_{i}>0.75$, and there are two stars previously considered nonmembers that have a nonzero membership in the mixture model. The first nonmember, DES J000104.89-594814.4, has ${ }_{p i}=0.49$ and was considered a nonmember previously due to the velocity offset and the offset in color from the Tuc III CMD. Because the velocity offset from Tuc III's velocity is small $\left(\sim 10 \mathrm{~km} \mathrm{~s}^{-1}\right)$ and it has a low metallicity, it has a nonzero membership in the mixture model. The second, DES J234949.16-602020.5, has ${ }_{p i}=0.73$ and was considered a nonmember due to the metal-rich template providing a better fit than the metal-poor template due to the large CaT EW. As the $\mathrm{S} / \mathrm{N}$ was too low for an accurate CaT EW measurement, only the velocity was considered in the 

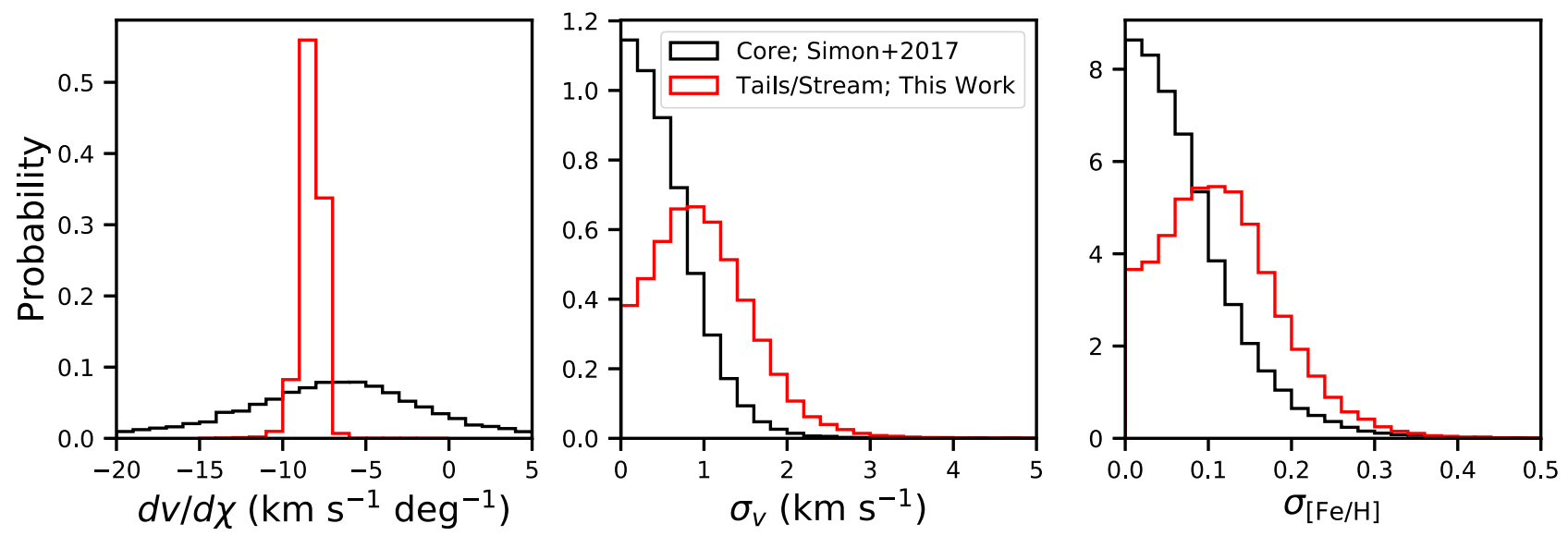

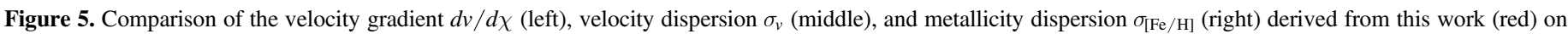

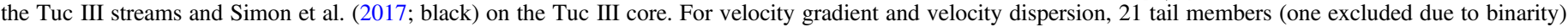
from this work were used. For metallicity dispersion, 16 tail+core RGB members from this work were used.

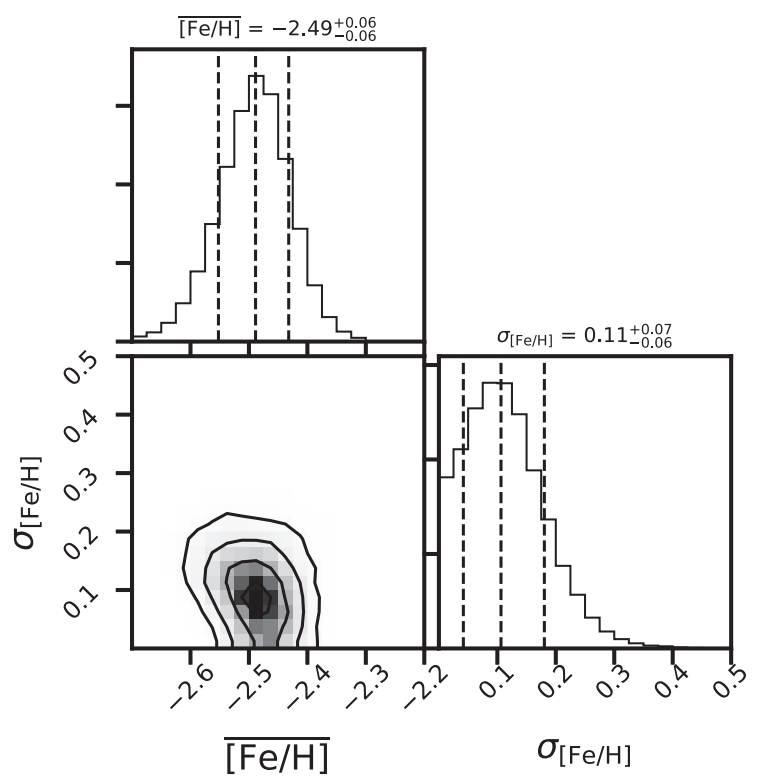

Figure 6. Posterior distribution of mean metallicity $\overline{[\mathrm{Fe} / \mathrm{H}]}$ and metallicity distribution $\sigma_{[\mathrm{Fe} / \mathrm{H}]}$ from $16 \mathrm{RGB}$ members.

mixture model, and the mixture model considers this star a probable member. The properties of Tuc III are not changed with respect to our results with the subjective analysis described in Section 3.1; we conclude that our determination of the Tuc III properties is robust. Overall, our Bayesian membership probabilities agree with the subjective membership.

We explored adding spatial information to the mixture model. We precomputed spatial probabilities based on a simple Gaussian model in stream latitude $B$ with stream width $\sigma_{w}=0.18$ (Shipp et al. 2018). We find that the spatial probability lowers the membership probabilities of the candidate stars at larger $B$ (especially the three members at $B \gtrsim 3 \sigma_{w}$ ), and therefore the overall membership decreases to $\sum p_{i}=26.4$. However, adding spatial information does not change the posterior distribution of the kinematic and chemical properties of the Tuc III stream, as shown in Figure 8.

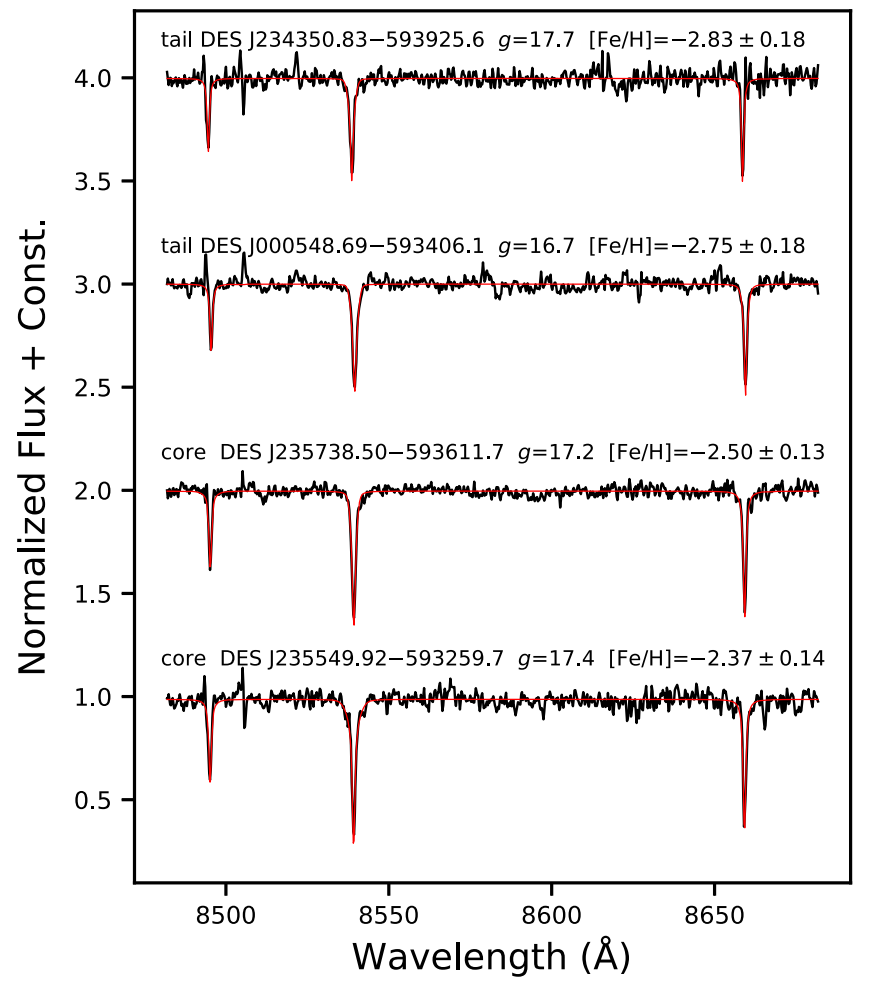

Figure 7. Spectra of four Tuc III stream members, two tail members, and two core members observed by AAT, shown with black lines. The red lines are the best-fit model for measuring the CaT EWs, as described in Section 2.3. The member stars are chosen so that they have similar brightnesses to minimize the surface gravity effect on CaT EWs. The two tail stars have smaller EWs and therefore are more metal-poor compared to the core stars at a similar brightness.

\section{Discussion}

\subsection{The Properties of the Tuc III Stream \\ 4.1.1. Density Variation along the Stream}

As shown in Figure 2, if we ignore the members confirmed by IMACS (which probes much deeper than AAT) and only focus on the 29 confirmed members from AAT, we notice obvious underdensities around $\Lambda \sim \pm 0^{\circ} .5$. As discussed in Section 3.1, the bright members with $g<19.5$ are mostly 


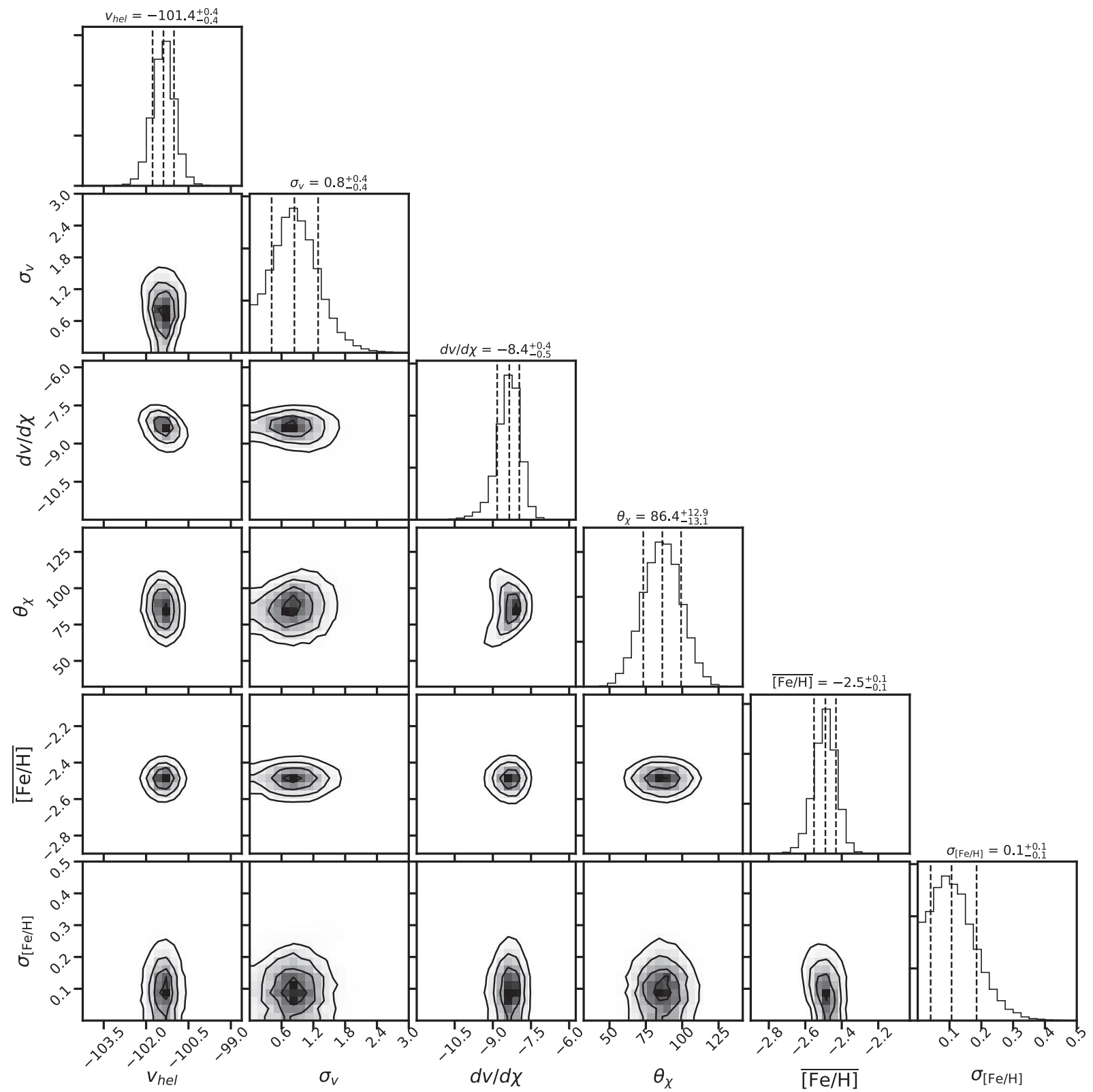

Figure 8. Posterior distributions of the Tuc III stream and MW mixture model from 552 stars in the three AAT fields. Only the Tuc III stream properties of the mixture model are displayed here. They are, from left to right, systemic velocity $\left(v_{\text {hel }}\right)$, velocity dispersion $\left(\sigma_{v}\right)$, velocity gradient $(d v / d \chi)$, position angle of the velocity gradient $\left(\theta_{\chi}\right)$, average metallicity $(\overline{[\mathrm{Fe} / \mathrm{H}]})$, and metallicity dispersion $\left(\sigma_{[\mathrm{Fe} / \mathrm{H}]}\right)$. The posteriors of the Tuc III properties in the mixture model are very similar to the results from the subjective membership selection.

identified within the fields of three AAT pointings. We therefore believe this nonuniform distribution of bright member stars is not a cause of observational bias. Though these underdensities could be a result of small-number statistics $(\sim 20$ tail stars from AAT), they may also just appear underdense relative to the epicyclic overdensities arising from tidal disruption (e.g., Küpper et al. 2008, 2010) that have been seen in the Pal 5 stream (e.g., Erkal et al. 2017; Küpper et al. 2017). Given the short amount of time needed to form a stream as long as Tuc III (see Erkal et al. 2018), the fact that no more wiggles (overdensity + underdensity) have been seen in these bright members might also reflect the fact that the progenitor has only had one pericentric passage (where the first tidal disruption happened). Additional modeling is needed to investigate the formation of the stream. Given the small sample with bright member stars, we suggest that the density variation along the stream longitude should be further investigated and verified with deeper photometry data.

\subsubsection{The Stream Orbit}

As shown in Figures 1 and 2, the RV of the Tuc III stream member stars decreases toward smaller R.A. $\left(\alpha_{2000}\right)$ or larger stream longitude $(\Lambda)$. Tuc III has a velocity of $v_{\mathrm{GSR}}=-195.2 \mathrm{~km} \mathrm{~s}^{-1}$ at $\Lambda=0$ and $d v_{\mathrm{GSR}} / d \Lambda=$ $-6.1 \mathrm{~km} \mathrm{~s}^{-1} \mathrm{deg}^{-1}$ in the Galactic standard of rest (GSR) frame. 
Therefore, the stream is moving toward the Galactic center; the west tail (or $\Lambda>0$ ) is the leading arm and moving faster toward us, and the east tail (or $\Lambda<0$ ) is the trailing arm and moving slower.

Furthermore, Shipp et al. (2018) reported that a distance gradient of $\frac{d(m-M)}{d \Lambda}=0.14 \pm 0.05 \mathrm{mag} \mathrm{deg}^{-1}$ was detected along the Tuc III stream, implying (given the stream's position relative to the Galactic center) that the Tuc III stream is on a radial orbit. The large velocity gradient measured in this work matches with the picture of the large distance gradient from photometry measurements. In fact, Tuc III is likely on a highly eccentric $(e \sim 0.9)$, inclined orbit with a pericenter of several kpc from the Galactic center, though this orbit largely depends on the mass of the Large Magellanic Cloud (LMC). We refer readers to Erkal et al. (2018) for more detailed modeling work on the orbit of the Tuc III stream that uses the stream track and distance measured from the DES photometry, as well as the velocity and velocity gradient from this work.

The orbit of the Tuc III stream will be further constrained by the proper motions of the stream, which will soon be measured by the upcoming Gaia DR2. We computed the expected precision of the proper motions with which the Tuc III stream will be measured using the spectroscopically confirmed members from this work (see details in Appendix B). The

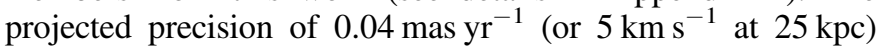
will place very tight constraints on the orbit of the Tuc III stream and further constraints on the mass of the LMC (see Erkal et al. 2018).

\subsubsection{The Nature of the Progenitor}

The nature of Tuc III is still under debate. Due to its low metallicity and size, Simon et al. (2017) tentatively suggested that Tuc III is the tidally stripped remnant of a dark matterdominated dwarf galaxy. Indeed, if the total stellar mass of the progenitor is the same as the Tuc III stream as measured by Shipp et al. (2018; i.e., $3.8 \times 10^{3} M_{\odot}$ ), it would lie directly on the metallicity-luminosity relation of dwarf galaxies (Kirby et al. 2013). However, recent work by Simpson (2018) found a similarly low metallicity for the faint globular cluster ESO 280SC06, further blurring the boundary between dwarf galaxies and star clusters.

Apart from the metallicity, the large size of Tuc III $\left(r_{h} \sim 44 \mathrm{pc}\right)$ relative to the globular cluster population is another piece of evidence favoring a dwarf galaxy origin. If the progenitor is a star cluster, the unusually large size would presumably be a consequence of tidal stripping. However, even though stripping plus observational biases can potentially inflate the size of faint star clusters (Contenta et al. 2017), the radius of Tuc III is still large enough to be difficult to explain. While it is known that compact globular clusters $(r \lesssim 5 \mathrm{pc}$ ) can survive very close encounters with the center of the MW (e.g., Sohn et al. 2018), comparable measurements are not available for low-mass dwarf galaxies or extended outer halo clusters. Theoretical modeling of objects on such orbits could provide additional clues to the nature of Tuc III. For future studies, it is also important to obtain better and/or additional velocity measurements in the inner region of the stream (i.e., $0^{\circ} 05<|\Lambda|<0$.5) to detect where the gradient starts and identify the location of the transition between the remaining progenitor and the tidal tails (see discussions in Section 3.2).
As discussed in Section 3.3, driven by the three most metal-poor stars near the two ends of the stream, we found a marginally larger metallicity dispersion for the Tuc III stream compared to the upper limit in the Tuc III core (Simon et al. 2017), suggesting a possible dwarf galaxy origin for the Tuc III stream. If the outer halo of the progenitor object was tidally stripped first, then seeing more metal-poor stars farther from the core (along the stream direction) indicates a possible metallicity gradient in the progenitor, where the metal-poor stars are less centrally concentrated than the metal-rich ones. Similar trends have been seen in other MW satellite galaxies but at larger stellar masses (see, e.g., Kirby et al. 2011). This could also explain why the metallicity dispersion of the Tuc III core is small despite its progenitor being a dwarf galaxy. We measure $\sigma_{[\mathrm{Fe} / \mathrm{H}]}=0.11_{-0.06}^{+0.07} \mathrm{dex}$; as Willman \& Strader (2012) concluded that $\sigma_{[\mathrm{Fe} / \mathrm{H}]}>0.2 \mathrm{dex}$ robustly diagnoses a dwarf galaxy, the metallicity dispersion does not definitely classify this object. If Tuc III is a dwarf galaxy, the small dispersion inferred here is likely the result of the following two causes. First, as a result of the low $\mathrm{S} / \mathrm{N}$ of the AAT spectra, the uncertainty on the CaT-derived $[\mathrm{Fe} / \mathrm{H}]$ of individual stars is relatively large (most members have $[\mathrm{Fe} / \mathrm{H}]$ uncertainties larger than the median metallicity dispersion of $0.11 \mathrm{dex}$ ). Therefore, despite a large metallicity range $(-3.0<[\mathrm{Fe} / \mathrm{H}]<-2.3)$, the metallicity dispersion is not completely resolved (i.e., it is still consistent with zero). The dispersion can be refined with better metallicity determinations with either high-resolution spectroscopic follow-up observations or perhaps higher-S/N CaT spectra. Second, the sample size of the most metal-poor population is small, i.e., only three members at $[\mathrm{Fe} / \mathrm{H}]<-2.7$. It is possible that the progenitor contained more metal-poor members at $[\mathrm{Fe} / \mathrm{H}]<-2.7$ but they were stripped first and are now outside the known extent of the stream. Testing the metallicity gradient hypothesis requires mapping the entire Tuc III stream, in particular seeking lower-metallicity stars that might be located near the ends of the stream.

Furthermore, the chemical abundance patterns of the member stars could help the classification. For example, light-element abundance correlations (e.g $\mathrm{Na}-\mathrm{O}, \mathrm{Na}-\mathrm{Al}$, $\mathrm{Mg}-\mathrm{Al}$ ) appear to be ubiquitous in star clusters (e.g., Bastian \& Lardo 2017; Johnson et al. 2017). Many confirmed Tuc III stream members from this work are bright enough for a detailed abundance analysis via high-resolution spectroscopic observations.

Furthermore, based on the width of the stream, Shipp et al. (2018) derived the progenitor mass to be $\sim 8 \times 10^{4} M_{\odot}$. If the stellar luminosity of $2.8 \times 10^{3} L_{\odot}\left(\right.$ or $\left.M_{v}=-3.8\right)$ reported in Shipp et al. (2018) is close to the total stellar luminosity of the progenitor, it would imply a mass-to-light ratio of $\sim 40 M_{\odot} / L_{\odot}$, indicating a possible dwarf galaxy classification, though most of the ultra-faint dwarf galaxies at a similar luminosity have a much larger mass-to-light ratio. ${ }^{53}$

\subsection{Comparison with Other Streams and Satellites}

\subsubsection{Palomar 5}

Of all the thin streams known so far, the tidal tails of the globular cluster Palomar 5 (Pal 5) are in many ways similar to

\footnotetext{
53 We also note most of the mass-to-light ratios are defined within the halflight radius, which is very different from how the progenitor mass was calculated based on the width of stream.
} 
the Tuc III tails-both have an identified unambiguous progenitor and a similar velocity dispersion (see below). First detected in SDSS (Odenkirchen et al. 2001), the stellar stream of the Palomar 5 globular cluster extends over at least $22^{\circ}$ (Ibata et al. 2016). A velocity gradient (in heliocentric frame) in the range $0.4-1.0 \mathrm{~km} \mathrm{~s}^{-1} \mathrm{deg}^{-1}$ was first detected by Odenkirchen et al. (2009) and later confirmed with larger data sets (Kuzma et al. 2015; Ibata et al. 2017). While at a similar heliocentric and Galactocentric distance, it is noteworthy that Tuc III possesses a velocity gradient $10 \times$ larger than that of Pal 5. Ibata et al. (2017) found that the stellar mass of the tidal tails is $3 \times$ the mass of the core, which is very similar to the Tuc III stream, though Tuc III appears shorter on the sky $\left(5^{\circ}\right.$ versus $\left.22^{\circ}\right)$, partially due to the projection from its orientation.

Though the progenitor of the Tuc III stream is more likely to be a dwarf galaxy based on the large range of metallicities in member stars, as discussed in Section 4.1.3, the velocity dispersion of the Tuc III tails is smaller than that of the Pal 5 stream (2.1 $\pm 0.4 \mathrm{~km} \mathrm{~s}^{-1}$; Kuzma et al. 2015). This low velocity dispersion of the Tuc III stream makes it a good target to search for stream density perturbations caused by close encounters with dark matter subhalos (e.g., Erkal \& Belokurov 2015b). The short length of the Tuc III stream, however, may imply that the stream formed recently, and therefore there might not have been enough time for dark matter subhalos to perturb the stream density. A more precise model of the stream will clarify the extent to which the stream is only apparently short due to it being aligned with our line of sight from the Sun. Even if the short length is only a projection effect, it will likely make it harder to search for gaps and wiggles along the stream. In addition, if there are density variations near the progenitor due to its secular disruption, these will need to be accounted for in the search for subhalos.

\subsubsection{Tidal Features Associated with Dwarf Galaxies}

The kinematics and morphology of the Tuc III tails leave no doubt that they are physically associated with the satellite and that the tidal stream contains a large velocity gradient. This result suggests that the observation of velocity gradients can be a good way to assess the dynamical state of dwarf galaxies (Piatek \& Pryor 1995). Below, we discuss other MW dwarfs that have been claimed to contain extratidal features and/or velocity gradients and compare them with the Tuc III stream.

Circumstantial photometric and kinematic evidence has been used to argue that several other dwarf galaxies are being tidally disrupted. For example, unusually high ellipticities (e.g., Hercules, Ursa Major II; Sand et al. 2009; Muñoz et al. 2010), irregular outer isophotes (e.g., Ursa Major I, Ursa Major II; Okamoto et al. 2008; Muñoz et al. 2010; although see Martin et al. 2008 regarding the significance of such features), extratidal substructures (e.g., Hercules; Sand et al. 2009), and kinematic substructure or velocity gradients (e.g., Coma Berenices, Hercules, Leo V, Ursa Major II; Simon \& Geha 2007; Adén et al. 2009; Collins et al. 2017) have been found in several satellites and interpreted as tidal features. It is important to keep in mind, however, that the common attribution of such features to tidal stripping is not borne out by simulations of the stripping process (Muñoz et al. 2008).

A prime example of a dwarf galaxy often suggested to be disrupting is Hercules. Many authors have considered its extremely elongated stellar distribution as evidence of tidal disruption (Belokurov et al. 2007; Coleman et al. 2007; Sand et al. 2009; Roderick et al. 2015). Extratidal stellar overdensities, especially along the major axis, have also been identified (Sand et al. 2009; Fabrizio et al. 2014; Roderick et al. 2015), and several RR Lyrae variables are located at large projected separations from the dwarf (Garling et al. 2018). Note that while multiple studies have detected stellar overdensities, many of them do not overlap. Hercules has also been claimed to contain a velocity gradient (Adén et al. 2009; Deason et al. 2012), but the statistical significance of the gradient is very low $(1.2 \sigma)$, and a much larger spectroscopic sample over a wider area would be needed to test its reality (similar to our results in the Tuc III core). Martin \& Jin (2010) argued that Hercules could be an unbound stellar stream resulting from the disruption of a dwarf galaxy. Küpper et al. (2017) suggested that Hercules is on a very eccentric orbit and that Hercules and any extra structure are perpendicular to the orbit.

Of the ultra-faint dwarf galaxies often cited as undergoing tidal disruption in the literature, Leo $\mathrm{V}$ is notable for its similarities to Tuc III. There is some evidence for tidal disruption based on the stellar distributions (Belokurov et al. 2008; de Jong et al. 2010; Sand et al. 2012), in particular the extended BHB population (Belokurov et al. 2008; Sand et al. 2012) and RR Lyrae stars (Medina et al. 2017). Leo V has a tentative velocity gradient (Collins et al. 2017) with roughly four times the magnitude of Tuc III $\left(\sim 80 \mathrm{~km} \mathrm{~s}^{-1} \mathrm{kpc}^{-1}\right)$, but it was measured with only eight stars over just $\sim 3^{\prime}$. Similarly, Walker et al. (2009) found two potential members at large radii $\left(r \approx 13^{\prime}\right)$ and argued that Leo $\mathrm{V}$ is losing mass. A velocity gradient and members at large radii could indicate stripping from Leo V. If Leo V is undergoing tidal disruption similar to Tuc III, the lack of apparent tails may be due to the large distance of the satellite. In this scenario, deeper observations of the main sequence might reveal an extended structure. As a note, the surface brightness of the Tuc III core is about $29 \mathrm{mag} \mathrm{arcsec}^{-2}$ (Drlica-Wagner et al. 2015), while the surface brightness of the tails is about 3 mag $\operatorname{arcsec}^{-2}$ fainter (Shipp et al. 2018).

A key difference between Tuc III and Hercules and Leo V is that the latter two are quite distant satellites of the MW ( $d>130 \mathrm{kpc}$; Musella et al. 2012; Sand et al. 2012), well beyond the region where the MW's tidal field could be causing stripping. If these objects are on very highly eccentric orbits, then they could have suffered significant tidal stripping at pericenter and are now located near apocenter (Küpper et al. 2017), but the required orbital eccentricities to bring them within a few kpc of the Galactic center at pericenter are extreme $(e>0.95)$. Moreover, numerical simulations indicate that dwarfs that are not completely disrupted should quickly return to equilibrium after a pericentric passage (e.g., Peñarrubia et al. 2008, 2009; Kazantzidis et al. 2011; Barber et al. 2015), in which case tidal features are not expected to be seen for objects that are currently far out in the halo of the MW. While it has been suggested that Leo V could be physically associated 
with its neighbor Leo IV and that a tidal interaction between the two is possible (de Jong et al. 2010), the mass required for the two systems to be gravitationally bound to one another is implausibly large for their luminosities (de Jong et al. 2010; Blaña et al. 2012).

Regarding the ultra-faint dwarf Segue 2, Kirby et al. (2013) argued that it was tidally stripped because it does not lie on the stellar mass-metallicity relationship. A similar argument was made for the Tuc III core (Simon et al. 2017). Including the stellar mass in the tidal tails will move Tuc III into agreement with other dwarf galaxies with respect to the stellar massmetallicity relationship. In contrast with Tuc III, there are no clear tidal features seen in the Segue 2 stellar distribution, even with data that reach the main sequence of Segue 2 (Belokurov et al. 2009).

\section{Selecting Metal-poor RGB Stars with DES Photometry}

Although halo substructures such as stellar streams and dwarf galaxies are most commonly identified by their MSTO stars, which dominate the total stellar counts of a system for typical survey depths, the spectroscopic follow-up observations for membership identification and kinematic measurements are mostly performed on RGB stars due to the faintness of the MSTO stars. In contrast to the hundreds to thousands of MSTO members, there are usually only a few dozen RGB members in a stellar stream or an ultra-faint dwarf galaxy. The efficiency of membership identification is extremely low due to a large amount of contamination from foreground stars in the MW disk and the low density of RGB members in these substructures, especially for stellar streams where the surface density is much lower compared to dwarfs. For example, of the 552 stars for which we obtained successful velocity measurements with the AAT observations, only 29 are members of the Tuc III stream. Fortunately, stellar streams and dwarf galaxies are mostly old and metal-poor populations; therefore, if the stellar metallicity can be roughly estimated using the photometry, the foreground contaminants could be largely removed, increasing the success rate of follow-up spectroscopy.

The broadband colors of stars are sensitive to their chemical composition. For example, many studies have found correlations between stellar colors and metallicities for $M$ dwarfs (Lépine 2008; Bochanski et al. 2013; Lépine et al. 2013; Li et al. 2014). For F/G stars, Ivezić et al. (2008) presented a correlation to estimate their effective temperature and metallicity using the position of the stars on the SDSS $u-g$ versus $g-r$ diagram. This method was based upon the traditional ultraviolet- (UV-) excess method or line-blanketing effect (see, e.g., Wildey et al. 1962; Sandage 1969). In other words, the metallicity of a subdwarf (i.e., metal-poor dwarf star) can be estimated with the difference between the star's $U-B$ color and what would be measured for a more metal-rich star with the same $B-V$ color, because more metal lines are present in the shorter wavelengths of a stellar spectrum. Unfortunately, the DES does not routinely use the DECam $u$ band. Here we study the locations of confirmed member stars in the Tuc III stream on the $g-r$ versus $r-i$ diagram. Thanks to the high photometric precision of the DES, the method described below can be used to improve the target selections for future spectroscopic observations seeking members in streams and dwarf galaxies.

We constructed a color-color diagram for the confirmed RGB members in the Tuc III stream in $g-r$ versus $r-i$ using the DES DR1 photometry when available. ${ }^{54} \mathrm{We}$ focus on the color range $0.4<g-r<0.8$ because this is the range for the RGB member stars in the Tuc III stream where the foreground contamination dominates.

As shown in the top left panel of Figure 9, all confirmed Tuc III members lie on one side of the empirical stellar locus, which was constructed as the median of stars in the dereddened DES photometry. Specifically, we select a sample of stars over the full survey footprint in regions with low interstellar extinction- $E(B-V)<0.015$ using the reddening map of (Schlegel et al. 1998) — that are unsaturated and measured with high $\mathrm{S} / \mathrm{N}$ in each of the $g, r, i$, and $z$ bands (e.g., $16 \lesssim r \lesssim 21)$. We bin these selected stars $(\sim 200,000)$ according to their $g-z$ color with $\sim 500$ stars in each of 429 bins and evaluate the median stellar colors in each bin. As the stream members are all metal-poor stars $([\mathrm{Fe} / \mathrm{H}]<-2)$, the clumping of the members suggests that the color of these RGB stars slightly depends on the metallicity of the star. We therefore plot the Dotter isochrones at age $=12.5 \mathrm{Gyr}$ and various metallicities in the top right panel of Figure 9 and find a clear indication that at a given $r-i$ color, metal-poor stars tend to be bluer in $g-r$. This trend is very similar to the $u-g$ versus $g-r$ diagram seen in Figure 2 of Ivezić et al. (2008). Similar to the UV excess in $U-B$ (or $u-g$ for SDSS), metalpoor stars also present a $g-r$ excess at a given $r-i$ color. We also plot the blanketing vectors (see, e.g., Sandage \& Eggen 1959; Wildey et al. 1962) in the $g-r$ versus $r-i$ diagram, which shows the shift in position from a metal-poor giant star to a metal-rich giant star at a constant temperature using the synthetic magnitude from the Dotter isochrones. For a more metal-rich star, the $g-r$ color gets redder and the $r-i$ color gets bluer.

This metallicity-dependent color will improve the efficiency of selecting stream or dwarf RGB candidate members by at least a factor of $50 \%$ (because all the member stars of the Tuc III stream are on one side of the stellar locus). This is extremely valuable for the spectroscopic follow-up program, where the number of fibers or slitlets of a multi-object spectrograph in one exposure is limited, and especially useful for stellar stream follow-up, where the member stars are sparsely populated and the foreground contamination from MW disk stars is relatively high. Furthermore, this colorcolor selection can remove foreground metal-rich disk stars and improve the detection significance for distant substructure searches (i.e., dwarfs and streams) using photometry alone, since in these distance structures $(d>200 \mathrm{kpc})$, only RGBs are brighter than the limiting magnitude of the imaging survey.

Note that from $[\mathrm{Fe} / \mathrm{H}]=-2.3$ to $[\mathrm{Fe} / \mathrm{H}]=-0.6$, the difference in $r-i$ is less than $0.05 \mathrm{mag}$. Therefore, this color difference cannot be revealed without the high-precision DES photometry (rms $<0.01 \mathrm{mag}$; see Burke et al. 2018). Furthermore, this color-color selection is most efficient for the brightest stars where the uncertainty from photon noise is negligible $(g<21)$. Fortunately, our spectroscopic targets are usually bright RGB stars, and therefore we can take advantage of the precise photometric calibration of the DES.

Following the trend discussed above, photometric metallicity could, in principle, be derived statistically for stars at

\footnotetext{
${ }^{54}$ Stars that have the Y2Q photometry in Table 1 are not included in Figure 9.
} 

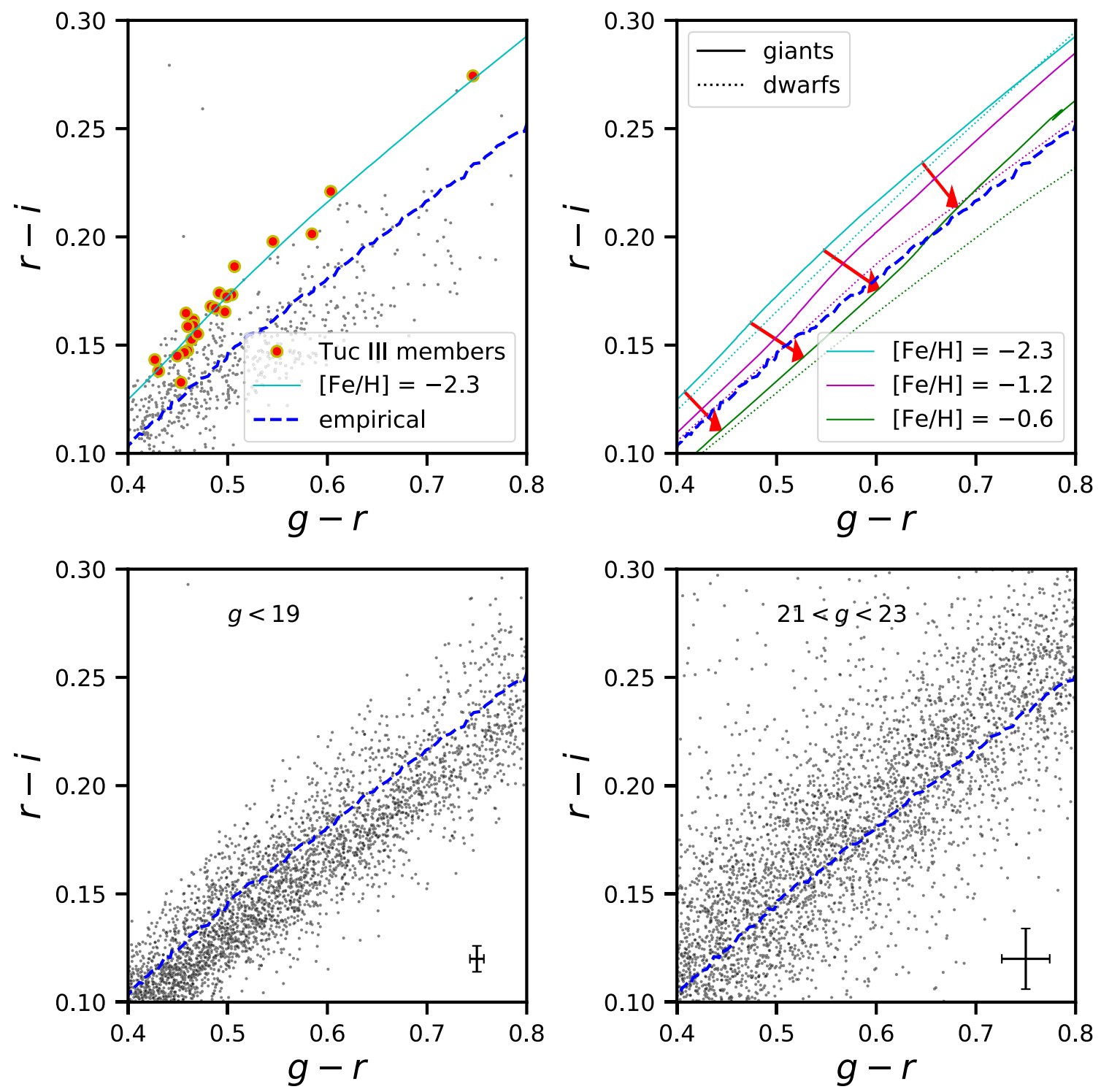

Figure 9. Top left: color-color diagram in $g-r$ vs. $r-i$ for the observed targets (gray dots) and confirmed members (red filled circles) in the Tuc III stream. The dashed blue line is an empirical stellar locus of DES photometry. All confirmed Tuc III members lie on one side of the empirical stellar locus and align well with a metal-poor isochrone at $[\mathrm{Fe} / \mathrm{H}]=-2.3$ using the synthetic magnitude from Dotter isochrones. Top right: synthetic magnitude from Dotter isochrones at age $=12.5 \mathrm{Gyr}$ and various metallicities, along with the empirical stellar locus as shown in the top left panel. The synthetic magnitude from the isochrone indicates a strong metallicity dependent on $g-r$ color at a given $r-i$ color. Also plotted (red vectors) are the isotherm lines (or blanketing vectors) for giant stars using the synthetic magnitude from the Dotter isochrones. At a given stellar temperature, the $g-r$ color increases (i.e., redder) and $r-i$ color decreases (i.e., bluer) from a metal-poor stellar population to a metal-rich stellar population. Bottom: stars in the field of the Tuc III stream with $g<19$ (bottom left) and $21<g<23$ (bottom right). The brighter (fainter) stellar bin is dominated by nearby disk stars (distant halo stars) and has more metal-rich (metal-poor) stars; thus, the majority of stars are below (above) the stellar locus. The high-precision DES photometry could provide a rough metallicity estimation of the red stars $(0.4<g-r<0.8)$ based on the $g-r$ vs. $r-i$ color of the stars and could further provide an estimation of the metallicity distribution function of the MW. We note that in the bottom right panel, due to the poorer star-galaxy separation at the fainter magnitude, we expect some galaxy contaminations whose colors are far away from the stellar locus. The median photometric uncertainty for each magnitude bin is also shown. The uncertainties are computed as a quadrature sum of the statistical uncertainty from the DES DR1 catalog (WAVG_MAGERR_PSF) and the systematic uncertainty as reported in DES DR1 (Abbott et al. 2018). In the brighter bin, the uncertainty is dominated by the systematic precision at 6-7 mmag (note that the spectroscopically confirmed Tuc III stream members have similar uncertainties). In the fainter bin, the uncertainty is dominated by the WAVG_MAGERR_PSF quantity with a median at $0.01-0.02 \mathrm{mag}$.

$0.4<g-r<0.8$ based on their colors and thereby derive the metallicity distribution function of the MW's disk and its stellar halo, though a more sophisticated calibration is needed to derive a more precise correlation between the metallicity of the stars and their positions in the $g-r$ versus $r-i$ diagram. As a proof of concept, we plot stars in the region of the Tuc III stream without any prior color-magnitude cut in the bottom panels of Figure 9. We selected stars in two groups, $g<19$ and $21<g<23$. The brighter stars are nearby and therefore dominated by metal-rich disk stars, and the fainter stars are more distant and therefore dominated by metal-poor halo stars. The brighter group has a majority of its stars below the empirical stellar locus (and therefore is more metal-rich), while the fainter group has more stars above the locus (and therefore is more metal-poor). We leave a more thorough study of this topic to a future paper. 


\section{Summary}

We report on a spectroscopic analysis of the Tuc III stream using the $2 \mathrm{dF}+\mathrm{AAOmega}$ spectrograph on the AAT and the IMACS spectrograph on the Magellan Baade Telescope. We identify, for the first time, 22 members in the tidal tails of Tuc III. Together with the 26 members in the Tuc III core previously confirmed by Simon et al. (2017), this study yields a total sample of 48 stars in the Tuc III stream. Using the tail members, we measured a large velocity gradient of $-8.0 \pm 0.4 \mathrm{~km} \mathrm{~s}^{-1} \mathrm{deg}^{-1}$ along the stream, consistent with the picture of the large distance gradient detected from the DES photometry (Shipp et al. 2018). This velocity gradient, many times larger than that of the Pal 5 stream, for instance, strongly suggests that Tuc III is on a radial orbit and passed close to the Galactic center. The membership and velocity information obtained in this work allows a detailed, precise orbit of Tuc III to be constructed (see Erkal et al. 2018) that will further our understanding of the mass distribution of our Galactic neighborhood, including the relative roles that dark matter, disk stars, and the LMC play in determining overall halo dynamics.

We found several more metal-poor member stars near the ends of the stream. These more metal-poor members farther from the center of Tuc III result in a slightly larger metallicity dispersion for the stream than that for the core alone, as derived in Simon et al. (2017), indicating that the progenitor of the Tuc III stream is likely to be a dwarf galaxy rather than a star cluster. However, the metallicity dispersion we found is still smaller than that of most dwarf galaxies at a similar luminosity. Additional metal-poor members farther from the center of the stream may be found in future observations if such a metallicity gradient is genuine.

We found that in a color-color diagram of $g-r$ versus $r-i$, all the member stars in the Tuc III stream are systematically redder in $r-i$ color (or bluer in $g-r$ ) than most nonmember stars. The high precision of DES photometry allows us to identify metal-poor stars photometrically. This metallicitydependent color offers a more efficient method for selecting metal-poor targets and will increase the efficiency of selecting stream members for future spectroscopic follow-up programs. Furthermore, the color-color selection can eliminate foreground metal-rich disk stars and improve the detection significance in finding dwarf galaxies and stellar streams using DES data (or other imaging surveys with a similar or better photometric precision).

TSL thanks Jo Bovy and Sergey Koposov for helpful conversations. JDS acknowledges support from the National Science Foundation under grant AST-1714873. ABP acknowledges generous support from the George P. and Cynthia Woods Institute for Fundamental Physics and Astronomy at Texas A\&M University. DE thanks Mark Gieles for helpful discussions. DE acknowledges financial support from the European Research Council under the European Union's Seventh Framework Programme (FP/2007-2013)/ERC Grant Agreement no. 308024. EB acknowledges financial support from the European Research Council (StG-335936). The authors thank the anonymous referee for a careful reading of the manuscript and providing useful comments.
Based on data obtained at Siding Spring Observatory via program A/2016A/26. We acknowledge the traditional owners of the land on which the AAT stands, the Gamilaraay people, and pay our respects to elders past and present.

This research has made use of NASA's Astrophysics Data System Bibliographic Services.

This paper has gone through internal review by the DES collaboration.

Funding for the DES Projects has been provided by the U.S. Department of Energy, the U.S. National Science Foundation, the Ministry of Science and Education of Spain, the Science and Technology Facilities Council of the United Kingdom, the Higher Education Funding Council for England, the National Center for Supercomputing Applications at the University of Illinois at Urbana-Champaign, the Kavli Institute of Cosmological Physics at the University of Chicago, the Center for Cosmology and Astro-Particle Physics at the Ohio State University, the Mitchell Institute for Fundamental Physics and Astronomy at Texas A\&M University, Financiadora de Estudos e Projetos, Fundação Carlos Chagas Filho de Amparo à Pesquisa do Estado do Rio de Janeiro, Conselho Nacional de Desenvolvimento Científico e Tecnológico, Ministério da Ciência, Tecnologia e Inovação, the Deutsche Forschungsgemeinschaft, and the Collaborating Institutions in the Dark Energy Survey.

The Collaborating Institutions are Argonne National Laboratory, the University of California at Santa Cruz, the University of Cambridge, Centro de Investigaciones Energéticas, Medioambientales y Tecnológicas-Madrid, the University of Chicago, University College London, the DESBrazil Consortium, the University of Edinburgh, the Eidgenössische Technische Hochschule (ETH) Zürich, Fermi National Accelerator Laboratory, the University of Illinois at Urbana-Champaign, the Institut de Ciències de l'Espai (IEEC/CSIC), the Institut de Física d'Altes Energies, Lawrence Berkeley National Laboratory, Ludwig-Maximilians Universität München and the associated Excellence Cluster Universe, the University of Michigan, the National Optical Astronomy Observatory, the University of Nottingham, The Ohio State University, the University of Pennsylvania, the University of Portsmouth, SLAC National Accelerator Laboratory, Stanford University, the University of Sussex, Texas A\&M University, and the OzDES Membership Consortium.

Based in part on observations at Cerro Tololo InterAmerican Observatory, National Optical Astronomy Observatory, which is operated by the Association of Universities for Research in Astronomy (AURA) under a cooperative agreement with the National Science Foundation.

The DES data management system is supported by the National Science Foundation under grant Numbers AST1138766 and AST-1536171. The DES participants from Spanish institutions are partially supported by MINECO under grants AYA2015-71825, ESP2015-66861, FPA2015-68048, SEV-2016-0588, SEV-2016-0597, and MDM-2015-0509, some of which include ERDF funds from the European Union. IFAE is partially funded by the CERCA program of the Generalitat de Catalunya. Research leading to these results has received funding from the European Research Council under the European Union's Seventh Framework Program (FP7/ 2007-2013), including ERC grant agreements 240672, 291329, and 306478. We acknowledge support from the Australian 
Research Council Centre of Excellence for All-sky Astrophysics (CAASTRO) through project number CE110001020 and the Brazilian Instituto Nacional de Ciência e Tecnologia (INCT) e-Universe (CNPq grant 465376/2014-2).

This manuscript has been authored by the Fermi Research Alliance, LLC, under contract No. DE-AC02-07CH11359 with the U.S. Department of Energy, Office of Science, Office of High Energy Physics. The United States Government retains and the publisher, by accepting the article for publication, acknowledges that the United States Government retains a nonexclusive, paid-up, irrevocable, worldwide license to publish or reproduce the published form of this manuscript, or allow others to do so, for United States Government purposes.

Facility: Anglo-Australian Telescope (AAOmega+2dF); Magellan Baade (IMACS).

Software: astropy (Astropy Collaboration et al. 2013), corner.py (Foreman-Mackey 2016), emcee (ForemanMackey et al. 2013), matplotlib (Hunter 2007), numpy (Van Der Walt et al. 2011), PyGaia (see footnote 55), scipy (Jones et al. 2001), ugali (Bechtol et al. 2015) (see footnote 50).

\section{Appendix A \\ Coordinate Transformation Matrix}

In Section 3.1, we described the transformation from celestial coordinates $(\alpha, \delta)$ to stream coordinates $(\Lambda, B)$ for the Tuc III stream using Euler angles $\left(\phi, \theta, \psi=264^{\circ} .23\right.$, $\left.120^{\circ} .29,267^{\circ} .51\right)$ so that the stream is roughly aligned along $B=0$ and the Tuc III core is at $\Lambda=0$. The transformation from $(\alpha, \delta)$ to $(\Lambda, B)$ is given by

$$
\begin{aligned}
& {\left[\begin{array}{c}
\cos (\Lambda) \cos (B) \\
\sin (\Lambda) \cos (B) \\
\sin (B)
\end{array}\right]=} \\
& {\left[\begin{array}{ccc}
0.505715 & -0.007435 & -0.862668 \\
-0.078639 & -0.996197 & -0.037514 \\
0.859109 & -0.086811 & 0.504377
\end{array}\right] \times\left[\begin{array}{c}
\cos (\alpha) \cos (\delta) \\
\sin (\alpha) \cos (\delta) \\
\sin (\delta)
\end{array}\right] .}
\end{aligned}
$$

\section{Appendix B}

\section{Expected Proper Motion Precision from GAIA DR2}

The 29 member stars confirmed by AAT are relatively bright $(g<20)$ and will soon have proper motion measurements from Gaia DR2. Here we estimate the expected precision of proper motion on the Tuc III stream from Gaia DR2 (Gaia Collaboration et al. 2016). We computed the expected proper motion uncertainties from Gaia DR2 for every star using the PyGaia package ${ }^{55}$ given the Gaia $G$-band magnitude and $V-I$ color of each star. We first convert the DES photometry to Gaia $G$-band photometry using the transformation equation reported in the Appendix of Abbott et al. (2018). Since $V-I$ color is unavailable for these stars, we replaced it with DES $g-i$ color instead. We note that there is a small offset between the two, but a shift of $0.1 \mathrm{mag}$ in $V-I$ will only cause a $0.5 \mu$ as $\mathrm{yr}^{-1}$ change in the proper motion for the stars at $G=20$. We therefore conclude that the effect of replacing $V-I$ with $g-r$ is minimal. Since proper motion errors scale like $t^{-1.5}$, where $t$ is the duration of the observations, we also scaled the error by a factor of $4.5 \times$ of what pygaia computes, taking into account that DR2 only includes the Gaia data from the first 22 months of the entire $5 \mathrm{yr}$ mission length. ${ }^{56}$ The projected proper motion uncertainty is roughly $0.1<0.2<0.5<1.0$ mas $\mathrm{yr}^{-1}$ for individual stars with $r$-band magnitudes of $16.1<17.3<18.6<19.6$

We then compute the weighted averaged uncertainty of the 29 member stars as the expected precision of the proper motion on the Tuc III stream, which is on the order of $\sim 0.04$ mas $\mathrm{yr}^{-1}$. We caution that this projected overall uncertainty is calculated by assuming that the uncertainty on each individual star is largely dominated by the statistical uncertainty and therefore the overall uncertainty will be reduced by averaging all of the measurements together. If the precision from Gaia is systematics-limited at this brightness, then the final overall uncertainty on Tuc III could be much larger.

We also note that this weighted average uncertainty is mainly determined by the six brightest RGB members, which each have projected proper motion uncertainties of $<0.2$ mas $y r^{-1}$. Recently, four RR Lyrae stars have been found (C. E. Martínez-Vázquez 2018, in preparation) along the Tuc III stream that are not in this spectroscopically confirmed sample. The RR Lyrae stars in Tuc III are relatively bright. Assuming a magnitude of $G \sim 17.5$ and $V-I \sim 0.2$, each RR Lyrae star will have a proper motion

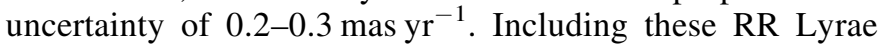
stars will further improve the precision of the stream proper motion.

\section{Appendix C \\ Potential Members of the Tuc III Stream}

During the velocity measurements, we found another 52 candidate members that suggest a tentative velocity in the range of $-140 \mathrm{~km} \mathrm{~s}^{-1}<v<-70 \mathrm{~km} \mathrm{~s}^{-1}$. However, the low $\mathrm{S} / \mathrm{Ns}(1 \lesssim v \lesssim 6)$ of the spectra did not pass the visual inspection in the fit. We did not include them in the analysis, but we list these stars and the best-fit velocity in Table 3 . We caution about the use of these measurements in RV (and therefore the uncertainties are not provided), but we suggest that these stars can be followed up spectroscopically with larger telescopes to verify their velocities and membership. Some of these stars are brighter than the limiting magnitude of Gaia DR2, and, accordingly, their membership status could be tested in the near future through precise proper motion measurements. 
Table 3

Potential Members in the Tuc III Stream

\begin{tabular}{|c|c|c|c|c|c|c|c|c|}
\hline ID & $\begin{array}{l}\alpha_{2000} \\
(\mathrm{deg})\end{array}$ & $\begin{array}{l}\delta_{2000} \\
(\mathrm{deg})\end{array}$ & $\begin{array}{c}g \\
(\mathrm{mag})\end{array}$ & $\begin{array}{c}r \\
(\mathrm{mag})\end{array}$ & Cat. & Inst. & $\mathrm{S} / \mathrm{N}$ & $\begin{array}{c}v \\
\left(\mathrm{~km} \mathrm{~s}^{-1}\right)\end{array}$ \\
\hline DES J233957.91-593718.3 & 354.99128 & -59.62174 & 19.392 & 18.950 & DR1 & AAT & 5.1 & -126.85 \\
\hline DES J234209.92-594724.9 & 355.54135 & -59.79024 & 19.806 & 19.398 & DR1 & AAT & 2.4 & -129.83 \\
\hline DES J234310.16-595556.6 & 355.79232 & -59.93240 & 20.956 & 20.758 & DR1 & AAT & 3.1 & -82.66 \\
\hline DES J234311.08-590221.5 & 355.79615 & -59.03932 & 19.330 & 18.851 & DR1 & AAT & 2.2 & -73.82 \\
\hline DES J234325.17-594435.1 & 355.85489 & -59.74308 & 18.763 & 18.320 & DR1 & AAT & 4.5 & -82.15 \\
\hline DES J234502.83-594108.5 & 356.26180 & -59.68569 & 20.696 & 20.437 & DR1 & AAT & 2.1 & -96.39 \\
\hline DES J234539.31-600534.8 & 356.41378 & -60.09300 & 20.413 & 20.165 & DR1 & AAT & 3.3 & -85.55 \\
\hline DES J234653.40-600737.8 & 356.72251 & -60.12717 & 19.906 & 19.502 & DR1 & AAT & 5.3 & -114.54 \\
\hline DES J234703.67-592837.7 & 356.76528 & -59.47714 & 19.933 & 19.527 & DR1 & AAT & 4.7 & -135.28 \\
\hline DES J234722.75-601040.8 & 356.84479 & -60.17799 & 20.271 & 19.968 & DR1 & AAT & 2.0 & -100.87 \\
\hline DES J234754.05-593615.2 & 356.97523 & -59.60422 & 20.103 & 19.714 & DR1 & AAT & 4.2 & -117.72 \\
\hline DES J234916.70-595028.3 & 357.31959 & -59.84118 & 20.457 & 20.231 & DR1 & AAT & 3.8 & -125.84 \\
\hline DES J234934.85-594127.9 & 357.39519 & -59.69107 & 20.718 & 20.501 & DR1 & AAT & 1.9 & -130.20 \\
\hline DES J234955.16-593738.0 & 357.47985 & -59.62722 & 20.374 & 20.128 & DR1 & AAT & 4.0 & -112.21 \\
\hline DES J235011.85-592433.7 & 357.54936 & -59.40936 & 20.308 & 20.025 & DR1 & AAT & 3.3 & -137.01 \\
\hline DES J235105.24-594437.3 & 357.77182 & -59.74369 & 20.372 & 20.098 & DR1 & AAT & 2.8 & -111.33 \\
\hline DES J235134.95-594124.6 & 357.89564 & -59.69016 & 19.846 & 19.411 & DR1 & AAT & 3.9 & -110.51 \\
\hline DES J235151.98-594056.9 & 357.96659 & -59.68247 & 19.806 & 19.377 & DR1 & AAT & 6.2 & -111.75 \\
\hline DES J235209.62-590450.5 & 358.04007 & -59.08070 & 19.844 & 19.411 & DR1 & AAT & 5.8 & -111.49 \\
\hline DES J235258.81-594111.3 & 358.24505 & -59.68646 & 20.553 & 20.337 & DR1 & AAT & 1.6 & -123.43 \\
\hline DES J235318.39-593243.8 & 358.32662 & -59.54549 & 20.422 & 20.179 & DR1 & IMACS & 4.5 & -100.10 \\
\hline DES J235328.33-593731.2 & 358.36804 & -59.62533 & 21.523 & 21.271 & DR1 & IMACS & 2.3 & -107.67 \\
\hline DES J235341.40-592600.9 & 358.42251 & -59.43358 & 19.796 & 19.329 & DR1 & AAT & 3.6 & -93.32 \\
\hline DES J235400.65-593255.5 & 358.50273 & -59.54876 & 20.414 & 20.195 & DR1 & IMACS & 5.5 & -99.64 \\
\hline DES J235712.70-600747.5 & 359.30291 & -60.12986 & 20.004 & 19.608 & DR1 & AAT & 3.1 & -116.45 \\
\hline DES J235901.96-592204.5 & 359.75815 & -59.36790 & 20.071 & 19.681 & DR1 & AAT & 2.1 & -87.20 \\
\hline DES J000101.57-593029.7 & 0.25654 & -59.50824 & 19.631 & 19.184 & DR1 & AAT & 4.5 & -96.29 \\
\hline DES J000105.45-592448.8 & 0.27272 & -59.41355 & 19.726 & 19.265 & DR1 & AAT & 4.3 & -100.99 \\
\hline DES J000133.88-595528.8 & 0.39116 & -59.92467 & 20.390 & 20.171 & DR1 & AAT & 2.2 & -106.77 \\
\hline DES J000336.20-595212.8 & 0.90082 & -59.87023 & 20.262 & 19.941 & DR1 & AAT & 1.8 & -100.49 \\
\hline DES J000400.30-593024.9 & 1.00126 & -59.50691 & 19.645 & 19.201 & DR1 & AAT & 4.5 & -94.87 \\
\hline DES J000505.18-593122.1 & 1.27159 & -59.52280 & 20.825 & 20.620 & DR1 & AAT & 2.0 & -117.23 \\
\hline DES J000731.43-593438.7 & 1.88097 & -59.57741 & 20.819 & 20.592 & DR1 & AAT & 5.4 & -104.87 \\
\hline DES J000826.30-593212.2 & 2.10958 & -59.53672 & 19.218 & 18.742 & DR1 & AAT & 6.7 & -93.81 \\
\hline DES J000841.53-591007.3 & 2.17302 & -59.16870 & 19.460 & 18.995 & DR1 & AAT & 2.7 & -86.19 \\
\hline DES J000902.34-594247.1 & 2.25975 & -59.71310 & 19.828 & 19.409 & DR1 & AAT & 4.7 & -71.10 \\
\hline
\end{tabular}

Note. Stars that are observed with AAT or IMACS and have measured RVs in the range of $-140 \mathrm{~km} \mathrm{~s}^{-1}<v<-70 \mathrm{~km} \mathrm{~s}^{-1}$, but the $\mathrm{S} / \mathrm{Ns}$ of the spectra are too low to obtain robust velocity measurements; therefore, these stars are not included in the analysis. The RVs should be used with caution. See details in Appendix C.

(This table is available in machine-readable form.) 


\section{ORCID iDs}

T. S. Li (1) https://orcid.org/0000-0002-9110-6163

K. Kuehn (당 https://orcid.org/0000-0003-0120-0808

B. Yanny (1) https://orcid.org/0000-0002-9541-2678

A. Drlica-Wagner (i) https://orcid.org/0000-0001-8251-933X

C. Lidman (1) https://orcid.org/0000-0003-1731-0497

N. Shipp (i) https://orcid.org/0000-0003-2497-091X

A. K. Vivas (1) https://orcid.org/0000-0003-4341-6172

A. R. Walker (i) https://orcid.org/0000-0002-7123-8943

R. H. Wechsler (1) https://orcid.org/0000-0003-2229-011X

J. Annis (i) https://orcid.org/0000-0002-0609-3987

D. Brooks (1) https://orcid.org/0000-0002-8458-5047

D. L. Burke (i) https://orcid.org/0000-0003-1866-1950

J. García-Bellido (1) https://orcid.org/0000-0002-9370-8360

D. W. Gerdes (i) https://orcid.org/0000-0001-6942-2736

D. Gruen (i) https://orcid.org/0000-0003-3270-7644

M. Soares-Santos (i) https://orcid.org/0000-0001-6082-8529

G. Tarle (i) https://orcid.org/0000-0003-1704-0781

\section{References}

Abbott, T. M. C., Abdalla, F. B., Allam, S., et al. 2018, arXiv:1801.03181 Adén, D., Wilkinson, M. I., Read, J. I., et al. 2009, ApJL, 706, L150 Alexander, P. E. R., \& Gieles, M. 2012, MNRAS, 422, 3415

Astropy Collaboration, Robitaille, T. P., Tollerud, E. J., et al. 2013, A\&A, 558, A33

Balbinot, E., Yanny, B., Li, T. S., et al. 2016, ApJ, 820, 58

Barber, C., Starkenburg, E., Navarro, J. F., \& McConnachie, A. W. 2015 , MNRAS, 447, 1112

Bastian, N., \& Lardo, C. 2017, arXiv:1712.01286

Baumgardt, H., Hut, P., \& Heggie, D. C. 2002, MNRAS, 336, 1069

Bechtol, K., Drlica-Wagner, A., Balbinot, E., et al. 2015, ApJ, 807, 50

Belokurov, V., Evans, N. W., Irwin, M. J., Hewett, P. C., \& Wilkinson, M. I. 2006a, ApJL, 637, L29

Belokurov, V., Walker, M. G., Evans, N. W., et al. 2008, ApJL, 686, L83

Belokurov, V., Walker, M. G., Evans, N. W., et al. 2009, MNRAS, 397, 1748

Belokurov, V., Zucker, D. B., Evans, N. W., et al. 2006b, ApJL, 642, L137

Belokurov, V., Zucker, D. B., Evans, N. W., et al. 2007, ApJ, 654, 897

Blaña, M., Fellhauer, M., \& Smith, R. 2012, A\&A, 542, A61

Bochanski, J. J., Savcheva, A., West, A. A., \& Hawley, S. L. 2013, AJ, 145, 40

Bonaca, A., Geha, M., Küpper, A. H. W., et al. 2014, ApJ, 795, 94

Bovy, J. 2014, ApJ, 795, 95

Bovy, J., Bahmanyar, A., Fritz, T. K., \& Kallivayalil, N. 2016, ApJ, 833, 31

Bovy, J., Erkal, D., \& Sanders, J. L. 2017, MNRAS, 466, 628

Bressan, A., Marigo, P., Girardi, L., et al. 2012, MNRAS, 427, 127

Bullock, J. S., \& Johnston, K. V. 2005, ApJ, 635, 931

Burke, D., Rykoff, E. S., Allam, S., et al. 2018, AJ, 155, 41

Carlberg, R. G. 2009, ApJL, 705, L223

Carlberg, R. G. 2016, ApJ, 820, 45

Carrera, R., Pancino, E., Gallart, C., \& del Pino, A. 2013, MNRAS, 434, 1681 Chabrier, G. 2001, ApJ, 554, 1274

Coleman, M. G., de Jong, J. T. A., Martin, N. F., et al. 2007, ApJL, 668, L43

Collins, M. L. M., Tollerud, E. J., Sand, D. J., et al. 2017, MNRAS, 467, 573

Contenta, F., Gieles, M., Balbinot, E., \& Collins, M. L. M. 2017, MNRAS, 466,1741

Cooper, M. C., Newman, J. A., Davis, M., Finkbeiner, D. P., \& Gerke, B. F. 2012, spec2d: DEEP2 DEIMOS Spectral Pipeline, Astrophysics Source Code Library, ascl:1203.003

Deason, A. J., Belokurov, V., Evans, N. W., Watkins, L. L., \& Fellhauer, M. 2012, MNRAS, 425, L101

de Jong, J. T. A., Martin, N. F., Rix, H.-W., et al. 2010, ApJ, 710, 1664

DES Collaboration 2005, arXiv:astro-ph/0510346

DES Collaboration 2016, MNRAS, 460, 1270

DES Collaboration 2017, arXiv:1708.01530

Dotter, A., Chaboyer, B., Jevremović, D., et al. 2008, ApJS, 178, 89

Dressler, A., Bigelow, B., Hare, T., et al. 2011, PASP, 123, 288

Dressler, A., Hare, T., Bigelow, B. C., \& Osip, D. J. 2006, Proc. SPIE, 6269 , $62690 \mathrm{~F}$

Drlica-Wagner, A., Bechtol, K., Rykoff, E. S., et al. 2015, ApJ, 813, 109

Erkal, D., \& Belokurov, V. 2015a, MNRAS, 450, 1136

Erkal, D., \& Belokurov, V. 2015b, MNRAS, 454, 3542
Erkal, D., Koposov, S. E., \& Belokurov, V. 2017, MNRAS, 470, 60

Erkal, D., Li, T. S., Koposov, S. E., et al. 2018, arXiv:1804.07762

Erkal, D., Sanders, J. L., \& Belokurov, V. 2016, MNRAS, 461, 1590

Fabrizio, M., Raimondo, G., Brocato, E., et al. 2014, A\&A, 570, A61

Feroz, F., \& Hobson, M. P. 2008, MNRAS, 384, 449

Feroz, F., Hobson, M. P., \& Bridges, M. 2009, MNRAS, 398, 1601

Fitzpatrick, E. L. 1999, PASP, 111, 63

Font, A. S., McCarthy, I. G., Crain, R. A., et al. 2011, MNRAS, 416, 2802

Foreman-Mackey, D. 2016, JOSS, 1, 24

Foreman-Mackey, D., Hogg, D. W., Lang, D., \& Goodman, J. 2013, PASP, 125,306

Gaia Collaboration, Prusti, T., de Bruijne, J. H. J., et al. 2016, A\&A, 595, A1

Garling, C., Willman, B., Sand, D. J., et al. 2018, ApJ, 852, 44

Hansen, T. T., Simon, J. D., Marshall, J. L., et al. 2017, ApJ, 838, 44

Hénon, M. 1961, AnAp, 24, 369

Hunter, J. D. 2007, CSE, 9, 90

Ibata, R. A., Lewis, G. F., Irwin, M. J., \& Quinn, T. 2002, MNRAS, 332, 915 Ibata, R. A., Lewis, G. F., \& Martin, N. F. 2016, ApJ, 819, 1

Ibata, R. A., Lewis, G. F., Thomas, G., Martin, N. F., \& Chapman, S. 2017, ApJ, 842, 120

Ivezić, Ž., Sesar, B., Jurić, M., et al. 2008, ApJ, 684, 287

Johnson, C. I., Caldwell, N., Rich, R. M., et al. 2017, ApJ, 842, 24

Johnston, K. V., Law, D. R., \& Majewski, S. R. 2005, ApJ, 619, 800

Johnston, K. V., Spergel, D. N., \& Haydn, C. 2002, ApJ, 570, 656

Jones, E., Oliphant, T., Peterson, P., et al. 2001, SciPy: Open source scientific tools for Python, http://www.scipy.org/

Kazantzidis, S., Łokas, E. L., Callegari, S., Mayer, L., \& Moustakas, L. A. 2011, ApJ, 726, 98

Kim, D., Jerjen, H., Mackey, D., Da Costa, G. S., \& Milone, A. P. 2015, ApJL, 804, L44

Kirby, E. N., Boylan-Kolchin, M., Cohen, J. G., et al. 2013, ApJ, 770, 16

Kirby, E. N., Lanfranchi, G. A., Simon, J. D., Cohen, J. G., \& Guhathakurta, P. 2011, ApJ, 727, 78

Küpper, A. H. W., Balbinot, E., Bonaca, A., et al. 2015, ApJ, 803, 80

Küpper, A. H. W., Johnston, K. V., Mieske, S., Collins, M. L. M., \& Tollerud, E. J. 2017, ApJ, 834, 112

Küpper, A. H. W., Kroupa, P., Baumgardt, H., \& Heggie, D. C. 2010 , MNRAS, 401, 105

Küpper, A. H. W., Lane, R. R., \& Heggie, D. C. 2012, MNRAS, 420, 2700

Küpper, A. H. W., MacLeod, A., \& Heggie, D. C. 2008, MNRAS, 387, 1248

Kuzma, P. B., Da Costa, G. S., Keller, S. C., \& Maunder, E. 2015, MNRAS, 446, 3297

Law, D. R., \& Majewski, S. R. 2010, ApJ, 714, 229

Lépine, S. 2008, AJ, 135, 2177

Lépine, S., Hilton, E. J., Mann, A. W., et al. 2013, AJ, 145, 102

Li, T., Marshall, J. L., Lépine, S., Williams, P., \& Chavez, J. 2014, AJ, 148, 60

Li, T. S., Simon, J. D., Drlica-Wagner, A., et al. 2017, ApJ, 838, 8

Li, T. S., Simon, J. D., Pace, A. B., et al. 2018, ApJ, 857, 145

Majewski, S. R., Skrutskie, M. F., Weinberg, M. D., \& Ostheimer, J. C. 2003, ApJ, 599, 1082

Martin, N. F., de Jong, J. T. A., \& Rix, H.-W. 2008, ApJ, 684, 1075

Martin, N. F., \& Jin, S. 2010, ApJ, 721, 1333

Martinez, G. D., Minor, Q. E., Bullock, J., et al. 2011, ApJ, 738, 55

Medina, G. E., Muñoz, R. R., Vivas, A. K., et al. 2017, ApJL, 845, L10

Muñoz, R. R., Geha, M., \& Willman, B. 2010, AJ, 140, 138

Muñoz, R. R., Majewski, S. R., \& Johnston, K. V. 2008, ApJ, 679, 346

Musella, I., Ripepi, V., Marconi, M., et al. 2012, ApJ, 756, 121

Newberg, H. J., \& Carlin, J. L. (ed.) 2016, Tidal Streams in the Local Group and Beyond (Vol. 420; Cham: Springer)

Newman, J. A., Cooper, M. C., Davis, M., et al. 2013, ApJS, 208, 5

Odenkirchen, M., Grebel, E. K., Kayser, A., Rix, H.-W., \& Dehnen, W. 2009, AJ, 137, 3378

Odenkirchen, M., Grebel, E. K., Rockosi, C. M., et al. 2001, ApJL, 548, L165

Oemler, A., Clardy, K., Kelson, D., Walth, G., \& Villanueva, E. 2017, COSMOS: Carnegie Observatories System for MultiObject Spectroscopy, Astrophysics Source Code Library, ascl:1705.001

Okamoto, S., Arimoto, N., Yamada, Y., \& Onodera, M. 2008, A\&A, 487, 103

Peebles, P. J. E. 1965, ApJ, 142, 1317

Peñarrubia, J., Navarro, J. F., \& McConnachie, A. W. 2008, ApJ, 673, 226

Peñarrubia, J., Navarro, J. F., McConnachie, A. W., \& Martin, N. F. 2009, ApJ, 698, 222

Piatek, S., \& Pryor, C. 1995, AJ, 109, 1071

Roderick, T. A., Jerjen, H., Mackey, A. D., \& Da Costa, G. S. 2015, ApJ, 804, 134

Sand, D. J., Olszewski, E. W., Willman, B., et al. 2009, ApJ, 704, 898

Sand, D. J., Strader, J., Willman, B., et al. 2012, ApJ, 756, 79 
Sandage, A. 1969, ApJ, 158, 1115

Sandage, A. R., \& Eggen, O. J. 1959, MNRAS, 119, 278

Schlafly, E. F., \& Finkbeiner, D. P. 2011, ApJ, 737, 103

Schlegel, D. J., Finkbeiner, D. P., \& Davis, M. 1998, ApJ, 500, 525

Searle, L., \& Zinn, R. 1978, ApJ, 225, 357

Sharp, R., Saunders, W., Smith, G., et al. 2006, Proc. SPIE, 6269, 62690G

Shipp, N., Drlica-Wagner, A., Balbinot, E., et al. 2018, ApJ, 862, 114

Simon, J. D., Drlica-Wagner, A., Li, T. S., et al. 2015, ApJ, 808, 95

Simon, J. D., \& Geha, M. 2007, ApJ, 670, 313

Simon, J. D., Li, T. S., Drlica-Wagner, A., et al. 2017, ApJ, 838, 11

Simpson, J. D. 2018, MNRAS, 477, 4565

Sohn, S. T., Watkins, L. L., Fardal, M. A., et al. 2018, ApJ, 862, 52
Spitzer, L. 1987, Dynamical Evolution of Globular Clusters (Princeton, NJ: Princeton Univ. Press)

Springel, V., Wang, J., Vogelsberger, M., et al. 2008, MNRAS, 391, 1685

Steinmetz, M., \& Navarro, J. F. 2002, NewA, 7, 155

Trotta, R. 2008, ConPh, 49, 71

Van Der Walt, S., Colbert, S. C., \& Varoquaux, G. 2011, CSE, 13, 22

Walker, M. G., Belokurov, V., Evans, N. W., et al. 2009, ApJL, 694, L144

Wildey, R. L., Burbidge, E. M., Sandage, A. R., \& Burbidge, G. R. 1962, ApJ, 135,94

Willman, B., \& Strader, J. 2012, AJ, 144, 76

Yoon, J. H., Johnston, K. V., \& Hogg, D. W. 2011, ApJ, 731, 58

Zacharias, N., Finch, C. T., Girard, T. M., et al. 2013, AJ, 145, 44 